Article

\title{
Stereoselective [4+2] Cycloaddition of Singlet Oxygen to Naphthalenes Controlled by Carbohydrates
}

\author{
Marcel Bauch, Werner Fudickar and Torsten Linker*D \\ Department of Chemistry, University of Potsdam, Karl-Liebknecht-Street 24-25, 14476 Golm, Germany; \\ marcel.potsdam@gmail.com (M.B.); wfudicka@uni-potsdam.de (W.F.) \\ * Correspondence: linker@uni-potsdam.de; Tel.: +49-331-977-5212
}

Citation: Bauch, M.; Fudickar, W.; Linker, T. Stereoselective [4+2] Cycloaddition of Singlet Oxygen to Naphthalenes Controlled by Carbohydrates. Molecules 2021, 26, 804. https://doi.org/10.3390/ molecules26040804

Academic Editor: Axel G. Griesbeck Received: 13 January 2021

Accepted: 2 February 2021

Published: 4 February 2021

Publisher's Note: MDPI stays neutral with regard to jurisdictional claims in published maps and institutional affiliations.

Copyright: (c) 2021 by the authors. Licensee MDPI, Basel, Switzerland. This article is an open access article distributed under the terms and conditions of the Creative Commons Attribution (CC BY) license (https:// creativecommons.org/licenses/by/ $4.0 /)$.

\begin{abstract}
Stereoselective reactions of singlet oxygen are of current interest. Since enantioselective photooxygenations have not been realized efficiently, auxiliary control is an attractive alternative. However, the obtained peroxides are often too labile for isolation or further transformations into enantiomerically pure products. Herein, we describe the oxidation of naphthalenes by singlet oxygen, where the face selectivity is controlled by carbohydrates for the first time. The synthesis of the precursors is easily achieved starting from naphthoquinone and a protected glucose derivative in only two steps. Photooxygenations proceed smoothly at low temperature, and we detected the corresponding endoperoxides as sole products by NMR. They are labile and can thermally react back to the parent naphthalenes and singlet oxygen. However, we could isolate and characterize two enantiomerically pure peroxides, which are sufficiently stable at room temperature. An interesting influence of substituents on the stereoselectivities of the photooxygenations has been found, ranging from 51:49 to up to 91:9 $d r$ (diastereomeric ratio). We explain this by a hindered rotation of the carbohydrate substituents, substantiated by a combination of NOESY measurements and theoretical calculations. Finally, we could transfer the chiral information from a pure endoperoxide to an epoxide, which was isolated after cleavage of the sugar chiral auxiliary in enantiomerically pure form.
\end{abstract}

Keywords: singlet oxygen; photooxygenation; naphthalenes; carbohydrates; stereoselectivity; auxiliary control; [4+2] cycloaddition

\section{Introduction}

Singlet oxygen $\left({ }^{1} \mathrm{O}_{2}\right)$ is an excited state of molecular oxygen and has been used for various oxidations, in biology, or medical applications [1]. Although the lifetime of this reactive species is short [2], depending on the solvent [3] or the biological environment [4], it can react with high regio- [5] and stereoselectivity [6]. It was the pioneering work of Adam, who discovered that $\mathrm{OH}$ groups can control the diastereoselectivity in ene reactions $[7,8]$ or $[4+2]$ cycloadditions of ${ }^{1} \mathrm{O}_{2}$ to naphthalenes $[9,10]$. Later on, the influence of other functional groups on photooxygenations has been investigated [6,11], and our group described the influence of acids and esters on ene reactions [12,13]. However, the enantioselective transfer of ${ }^{1} \mathrm{O}_{2}$ is still challenging, because of its short lifetime. Although the $\alpha$-hydroxylation of aldehydes and ketones was realized by organocatalysis with moderate to good ee (enantiomeric excess) [14-16], ene reactions or cycloadditions proceed less successfully. Thus, asymmetrically modified zeolites [17], chiral salts [18], or chiral sensitizers [19] afforded only low stereoselectivities. Best results with up to $90 \%$ ee have been obtained in photooxygenations of 2-pyridones in the presence of chiral bicyclic lactams, which, however, had to be used in excess [20].

Another possibility to control the face-selectivity of singlet oxygen attack are chiral auxiliaries. First attempts with oxazolines gave no selectivity [21], but menthol derivatives [22] or tartrates [23] showed moderate to good induction. Best results have been obtained with oxazolidines [24], sultams [25], and especially enecarbamates [26], all developed in the 
group of Adam [27]. However, to the best of our knowledge, carbohydrates have not served as chiral auxiliaries for the stereoselective transfer of ${ }^{1} \mathrm{O}_{2}$ until now. Only sugar-furans have been photooxygenated, but the intermediary formed peroxides rearrange thermally and cannot be isolated [28-30].

Based on our experience in carbohydrate chemistry [31-33] and acene oxidations [34-37], we became interested in a combination of both research topics and in sugar-substituted naphthalenes 1 (Scheme 1). The advantage of such arenes is that they react with ${ }^{1} \mathrm{O}_{2}$ by a [4+2] cycloaddition in high yields to endoperoxides (EPOs) 2, as pointed out with simple alkyl- [38] and methoxy-substituted [39] systems 1a $\left(\mathrm{R}^{1}=\mathrm{Alk}, \mathrm{OMe}\right)$. Furthermore, the EPOs 2a reconvert quantitatively to the parent naphthalene 1a under thermal conditions and under release of singlet oxygen [40-42]. We investigated the influence of substituents $\mathrm{R}^{2}$ on the kinetics of such reactions [43] and could establish an intramolecular transfer of ${ }^{1} \mathrm{O}_{2}$ [44]. However, carbohydrate-substituted naphthalenes $\mathbf{1} \mathbf{b}-\mathbf{f}$ were hitherto unknown, but will generate new stereogenic centers during the oxidation (Scheme 1). Herein, we describe the synthesis of such compounds, their photooxygenation, isolation of peroxides, release of singlet oxygen, and a transformation into an enantiomerically pure epoxide.

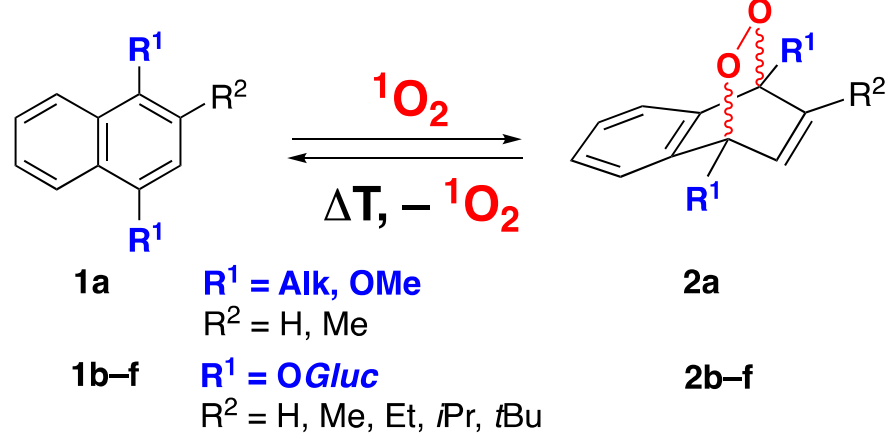

Scheme 1. Reversible reaction of naphthalenes 1 with singlet oxygen to endoperoxides 2.

\section{Results and Discussion}

The synthesis of carbohydrate-substituted naphthalenes $\mathbf{1 b}$-f started from 1,4-naphthoquinone (3b), an inexpensive and easily available precursor. Addition of alkyl radicals, generated by decarboxylation of acids 4 , afforded substituted naphthoquinones $3 \mathbf{c}-\mathbf{f}$ in moderate to good yields, in analogy to literature (Scheme 2) [45]. In situ reduction with sodium dithionite [46] gave the corresponding 1,4-dihydroxynaphthalenes 5, which were directly used for the next step without purification, because of their oxidation lability. Finally, Schmidt's trichloroacetimidate 6 [47,48] gave best results for glycosylations, and the hitherto unknown glucose-substituted naphthalenes $\mathbf{1 b} \mathbf{-} \mathbf{f}$ were isolated in good yields as single $\beta$-anomers in analytically pure form.

We started the photooxygenations with unsubstituted naphthalene $\mathbf{1 b}(\mathrm{R}=\mathrm{H})$. Thus, substrate $\mathbf{1 b}(123 \mathrm{mg}, 0.15 \mathrm{mmol})$ and sensitizer tetraphenylporphyrin (TPP, $1.0 \mathrm{mg})$ were dissolved in $\mathrm{CD}_{2} \mathrm{Cl}_{2}(3.0 \mathrm{~mL})$ on an NMR scale. Oxygen was slowly bubbled through the solution, which was irradiated externally with a sodium lamp (400 W) at room temperature. The progress of the reaction was followed by TLC and NMR (300 MHz, measurements for every $60 \mathrm{~min}$ ), however, even after $6 \mathrm{~h}$, no conversion was observed (Table 1, entry 1). This can be explained by a steric hindrance of the carbohydrate substituents, or by a reversible reaction of ${ }^{1} \mathrm{O}_{2}$ with naphthalenes [40,41], which is especially fast with alkoxy groups [39]. Indeed, we repeated the reaction at $-70^{\circ} \mathrm{C}$ and obtained full conversion after $2 \mathrm{~h}$ and the sole formation of one endoperoxide (EPO) $\mathbf{2 b}$, determined by NMR (500 MHz) at the same temperature (entry 2, for detailed NMR spectra see Supplementary Materials). The fact that the reaction is completely reversible was confirmed by thermolysis at different temperatures and by measurement of the reappearing naphthalene UV absorption band, which provided kinetic data. We determined a half lifetime of $2 \mathrm{~min}$ at $20{ }^{\circ} \mathrm{C}$, explaining why no conversion was observed at room temperature. Furthermore, at least $68 \%$ of 
the oxygen released during thermolysis was in its singlet state, which we proved by trapping with tetramethylethylene, and which is in accordance to simple naphthalene EPOs [40]. However, first attempts to transfer ${ }^{1} \mathrm{O}_{2}$ even enantioselectively to a prochiral acceptor failed.<smiles>CC1=CC(=O)c2ccccc2C1=O</smiles>

3b<smiles></smiles>
$\left(\mathrm{NH}_{4}\right)_{2} \mathrm{~S}_{2} \mathrm{O}_{8}$ $\mathrm{CH}_{3} \mathrm{CN} / \mathrm{H}_{2} \mathrm{O}$ $80^{\circ} \mathrm{C}, 4 \mathrm{~h}$<smiles>CC1=CC(=O)c2ccccc2C1=O</smiles>

$3 c \mathrm{R}=\mathrm{Me}(41 \%)$ $3 d \mathrm{~d}=\mathrm{Et}(50 \%)$ 3e $\mathrm{R}=\operatorname{IPr}(64 \%)$ 3f $\mathrm{R}=\mathrm{tBu}(73 \%)$<smiles>[R]c1cc(OC)c2ccccc2c1OC(C)=O</smiles>

$$
\begin{array}{ll}
\text { 1b } R=H & (76 \%) \\
\text { 1c } R=M e & (72 \%) \\
\text { 1d } R=E t & (74 \%) \\
\text { 1e } R=\operatorname{Pr} & (78 \%) \\
\text { 1f } R=t B u & (71 \%)
\end{array}
$$

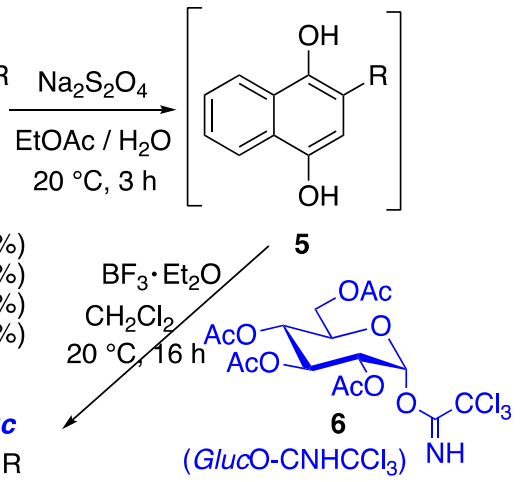

\begin{tabular}{|c|c|c|c|c|c|}
\hline Entry & Naphthalene 1 & $\mathbf{R}$ & $\begin{array}{l}\text { Temperature, } \\
\text { Conversion }^{2}\end{array}$ & $t_{1 / 2}\left(20{ }^{\circ} \mathrm{C}\right) 2^{3}$ & $d r 1 S, 4 R-2: 1 R, 4 S-2^{2}$ \\
\hline 1 & $1 b$ & $\mathrm{H}$ & $20^{\circ} \mathrm{C},<5 \%$ & $2 \mathrm{~min}$ & - \\
\hline 2 & $1 b$ & $\mathrm{H}$ & $-70{ }^{\circ} \mathrm{C},>98 \%$ & $2 \mathrm{~min}$ & - \\
\hline 3 & 1c & $\mathrm{Me}$ & $-70{ }^{\circ} \mathrm{C},>98 \%$ & $7 \mathrm{~min}$ & $90: 10$ \\
\hline 4 & 1d & Et & $-70{ }^{\circ} \mathrm{C},>98 \%$ & $9 \mathrm{~min}$ & $91: 9$ \\
\hline 5 & 1e & $i \operatorname{Pr}$ & $-70{ }^{\circ} \mathrm{C},>98 \%$ & $48 \mathrm{~min}$ & $89: 11$ \\
\hline 6 & 1f & $t \mathrm{Bu}$ & $-70{ }^{\circ} \mathrm{C},>98 \%$ & $>4 \mathrm{~h}^{4}$ & $51: 49^{5}$ \\
\hline
\end{tabular}

(GlucO-CNHCCl ${ }_{3}$ NH

Scheme 2. Synthesis of carbohydrate-substituted naphthalenes $\mathbf{1 b}-\mathbf{f}$ from naphthoquinone $\mathbf{3 b}$.

Table 1. Photooxygenation of naphthalenes $\mathbf{1} \mathbf{b}-\mathbf{f}$ to endoperoxides $\mathbf{2} \mathbf{b}-\mathbf{f}^{1}$.

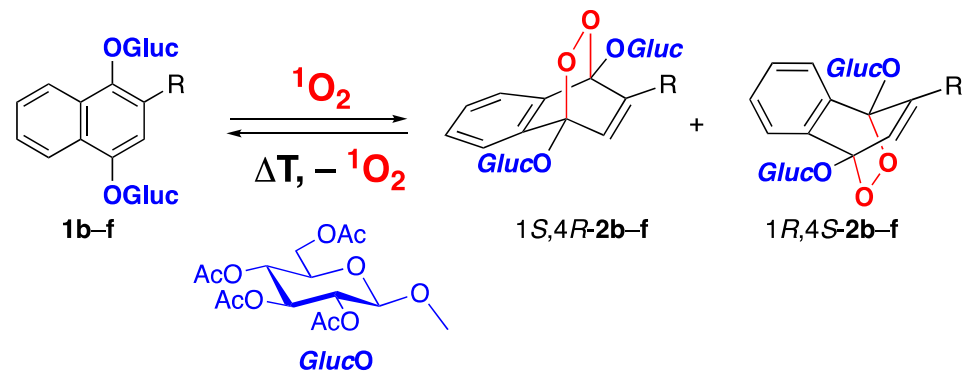

${ }^{1}$ Reactions were performed on a $0.15 \mathrm{mmol}$ NMR scale. For procedure and conditions, see Materials and Methods ${ }^{2}$ Determined by NMR $(500 \mathrm{MHz})$ of the crude products. ${ }^{3}$ Determined by UV measurements. ${ }^{4}$ For 77 min at $60{ }^{\circ} \mathrm{C} .{ }^{5}$ On a larger $1.0 \mathrm{mmol}$ scale, diastereomeric EPOs $2 \mathrm{f}$ could be separated by column chromatography and isolated in $46 \%$ and $41 \%$ in analytically pure form.

After the successful oxidation of naphthalene $\mathbf{1 b}$ (Table 1, entry 2), we next investigated the photooxygenations of the alkyl-substituted derivatives $\mathbf{1} \mathbf{c}-\mathbf{f}$ under the same conditions (entries 3-6). For all substrates, the conversion was complete after $2 \mathrm{~h}$, and we observed EPOs $\mathbf{2} \mathbf{c}-\mathbf{f}$ as sole products in the NMR of the crude reaction mixture at low temperature (for detailed NMR spectra, see Supplementary Materials). Again, thermolysis of all EPOs proceeded completely reversible, and the parent naphthalenes 1c-f were reisolated quantitatively. However, the half lifetimes differ depending on the substituents $\mathrm{R}$. Thus, the $t \mathrm{Bu}$ derivative $\mathbf{2} \mathbf{f}$ was stable at room temperature for several hours and the 
products could be isolated in analytically pure form by column chromatography (entry 6). The higher stability of EPOs with larger substituents is in accordance to our previous studies with achiral naphthalenes [43].

Besides the remarkable thermal stability of EPOs 2, we found an interesting influence of the substituents on the stereoselectivity of the photooxygenations (Table 1). For naphthalene $\mathbf{1 b}$, the two arene planes are identical, and only one EPO $\mathbf{2 b}$ can be formed (entry 2 ). However, for all other substrates $\mathbf{1} \mathbf{c}-\mathbf{f}$, singlet oxygen can attack from two different faces, affording two diastereomers $1 S, 4 R-\mathbf{2}$ and $1 R, 4 S-2$. Indeed, naphthalenes $1 \mathbf{c}-\mathbf{e}$ reacted with high stereoselectivities of about 9:1 to the corresponding EPOs 2c-e (entries 3-5). Surprisingly, the selectivity drops with $t$ Bu-substituted naphthalene $\mathbf{1 f}$ completely, and EPOs $2 \mathbf{f}$ are formed almost as 1:1 mixture (Table 1, entry 6). On the other hand, the diastereomers could be easily separated by column chromatography, characterized in pure form, and used for further transformations (see below). As the glucose substituents provide the stereochemical information, their steric and electrostatic repulsion of ${ }^{1} \mathrm{O}_{2}$ must be responsible for the selectivities, and thus, our concept of a carbohydrate-auxiliary-controlled photooxygenation has been realized.

The thermally labile peroxides $\mathbf{2} \mathbf{c}-\mathbf{e}$ could not be separated or isolated by column chromatography, but the $d r$ was unequivocally determined from the ${ }^{1}$ NMRs of the crude products at low temperature (Figure 1 for selected chemical shifts, see Supplementary Materials for complete spectra). Although carbohydrate signals overlap, arene and double bond protons give distinctive signals. For the single isomer $\mathbf{2} \mathbf{b}$, we obtained only one signal set with the protons at the double bond giving two doublets at 6.94 and $7.16 \mathrm{ppm}$. For EPOs $\mathbf{2} \mathbf{c}-\mathbf{f}$, the two diastereomers $1 S, 4 R-\mathbf{2}$ and $1 R, 4 S-\mathbf{2}$ exhibit two different signal sets, i.e., the major isomer as marked red and the minor isomer as marked green (Figure $1 \mathrm{~b}-\mathrm{e}$ ). Characteristic is the double doublet at $7.9 \mathrm{ppm}$ for the major isomers, which is assigned as $\mathrm{H}^{8}$ and speaks for its close proximity to the carbohydrate ring ortho to the substituent R. On the other hand, this proton has a chemical shift of $7.6 \mathrm{ppm}$ for the minor isomers (Figure 1, green), which is a remarkable difference.

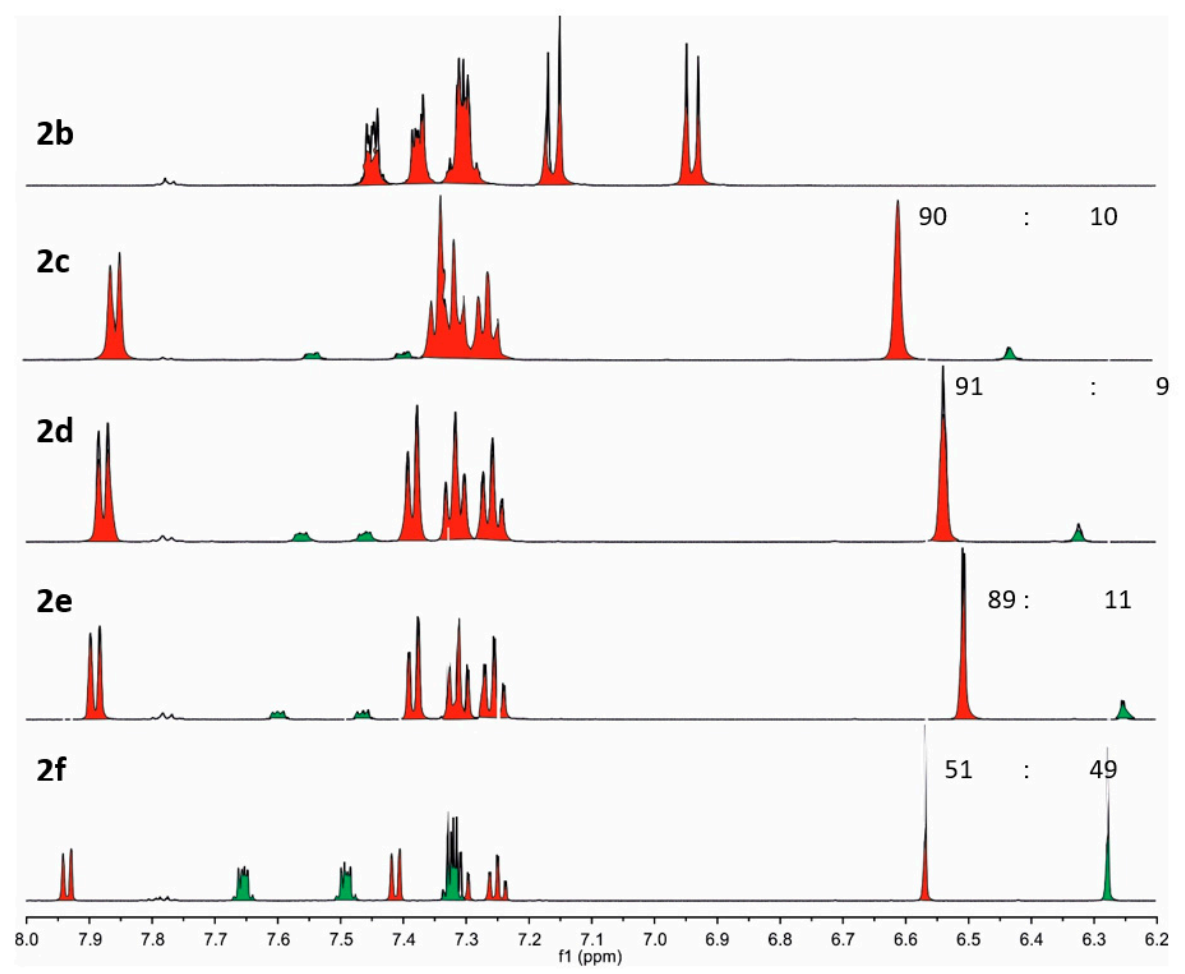

Figure 1. ${ }^{1} \mathrm{H}-\mathrm{NMR}$ spectra $(500 \mathrm{MHz}, 6-8 \mathrm{ppm})$ of the crude photooxygenation products of carbohydrate-substituted naphthalenes $\mathbf{2} \mathbf{b}-\mathbf{f}$ (major isomer red, minor isomer green). 
To understand this behavior, to determine the absolute configurations, and to find a rationale for the stereoselectivities, we analyzed the two diastereomeric EPOs $\mathbf{2} \mathbf{f}$, which we isolated in analytically pure form, in more detail by a combination of analytical and theoretical methods. Thus, we measured their NOESY spectra (Supplementary Materials), which gave distinctive cross-peaks (Figure 2). For both isomers, the anomeric proton of the carbohydrate in the 4-position is in close proximity to $\mathrm{H}^{3}$ and, therefore, to the double bond (marked green). On the other hand, only EPO 1S,4R-2f shows an NOE between the 6-OAc group of the sugar in the 1-position and $\mathrm{H}^{7}$ (marked blue), which is located far away in the benzene ring. Thus, this carbohydrate is pushed away by the sterically demanding $t \mathrm{Bu}$ group under the arene ring. Additionally, this explains the downfield shift of $\mathrm{H}^{8}$ to 8 ppm for all main isomers $1 S, 4 R-\mathbf{2} \mathbf{c}-\mathbf{f}$, resulting from the ring-oxygen of the carbohydrate. In contrast, in isomer EPO $1 R, 4 S-2 \mathbf{f}, \mathrm{H}^{8}$ shows a normal shift of $7.6 \mathrm{ppm}$ and an NOE is only observed to the 2-OAc and not to the 6-OAc group (marked orange). In summary, we propose for the main isomers a conformation with the carbohydrate in the 1-position far away from the peroxide bridge, whereas for the minor isomer, this sugar is in close proximity to the newly formed stereocenter.
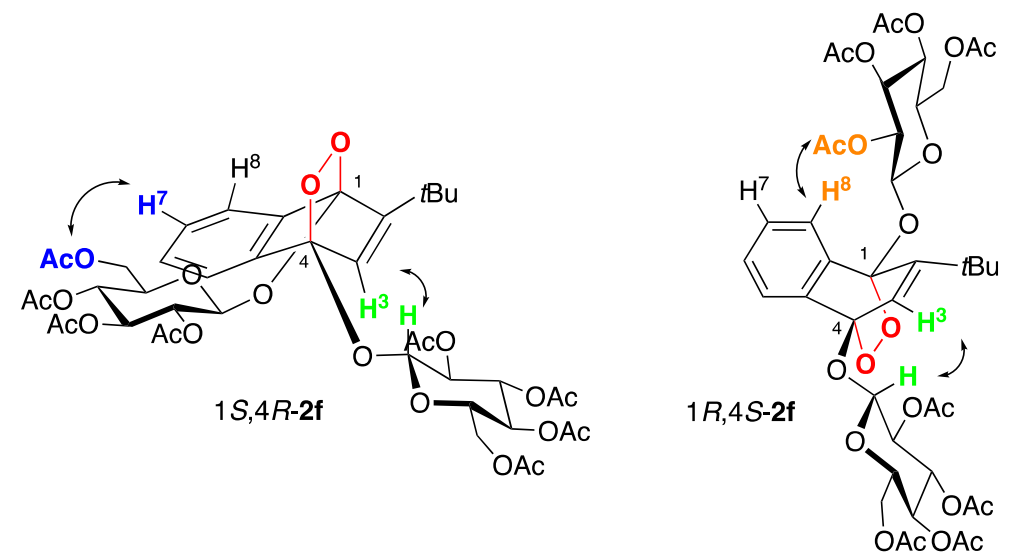

Figure 2. Characteristic NOE interactions in EPOs $1 S, 4 R-\mathbf{2 f}$ and $1 R, 4 S-\mathbf{2 f}$.

To further support the observed NOESY interactions, we conducted theoretical calculations at the B3LYP /6-31G* level (for details see Supplementary Materials). Thus, structures for both diastereomeric EPOs $1 S, 4 R-\mathbf{2 f}$ and $1 R, 4 S-\mathbf{2} \mathbf{f}$ were minimized (Figure 3). Indeed, for the main isomer, we find a close proximity of the 6-OAc group of the sugar in the 1-position and $\mathrm{H}^{7}$, which nicely is in accordance to the NOESY measurements (Figure 2). On the other hand, in EPO 1R,4S-2f, both carbohydrates are located in the plane of the peroxide bridge, again in good correlation to the NMR spectra.

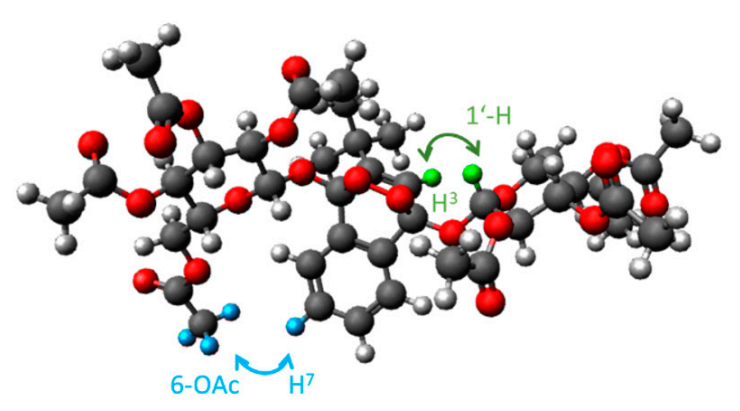

$1 S, 4 R-\mathbf{2 f}$

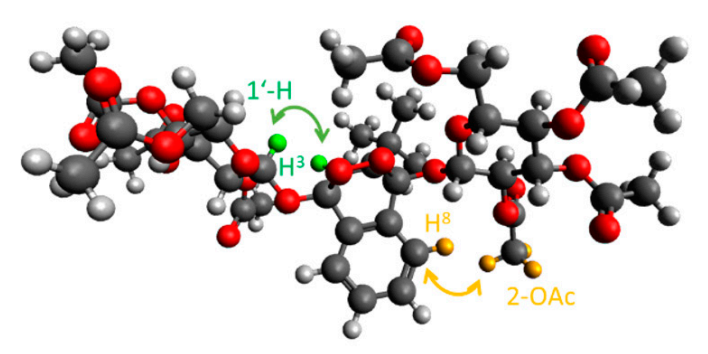

$1 R, 4 S-\mathbf{2 f}$

Figure 3. Calculated energetically minimized structures of EPOs $1 S, 4 R-\mathbf{2 f}$ and $1 R, 4 S-\mathbf{2 f}$. Carbon atoms are marked black; oxygen atoms are marked red; hydrogen atoms are marked grey; blue, green, and yellow indicate the distinct NOE interactions (compare Figure 2). 
Our structural assignment of the configurations and conformations of EPOs 2 might give an explanation for the observed stereoselectivities and influence of substituents as well (Figure 4). Thus, for starting materials $\mathbf{1} \mathbf{c}-\mathbf{f}$, the carbohydrate in the 1-position is pushed away by the substituent $\mathrm{R}$, resulting in a downfield shift of $\mathrm{H}^{8}$ (see NMR data in the section Materials and Methods). The second carbohydrate in the 4-position is much closer to the reacting naphthalene ring and, therefore, shields the si face. Thus, singlet oxygen has to attack from the opposite face, passing by the R group. Although the discussion of rotamers for the explanation of stereoselectivities is a bit speculative, our mechanistic proposal nicely fits to the observed influence of substituents. For naphthalene $\mathbf{1 b}(\mathrm{R}=\mathrm{H})$, no selectivity can result, but the photooxygenation of isomers $1 \mathrm{c}-\mathbf{e}(\mathrm{R}=\mathrm{Me}, \mathrm{Et}, \mathrm{iPr})$ provide good stereoselectivities (see Table 1$)$. Only for starting material $1 \mathrm{f}(\mathrm{R}=t \mathrm{Bu})$, the selectivity drops remarkably, because ${ }^{1} \mathrm{O}_{2}$ has to pass by this sterically demanding group, and therefore, both faces are shielded equally. The fact that the $t \mathrm{Bu}(\mathbf{1 f})$ and $i \operatorname{Pr}(\mathbf{1 e})$ derivatives react with such different selectivity is surprising, because their steric substituent constants are in the same range [49]. We explain this again by hindered rotations due to interactions with the carbohydrates. Thus, for $\mathrm{R}=i \mathrm{Pr}$, the two methyl groups may point away from the sugar in the 1-position, whereas for $\mathrm{R}=t \mathrm{Bu}$, one methyl group shields the upper plane (Figure 4). The fact that a $t \mathrm{Bu}$ group gives remarkably different stereoselectivities than an $i$ Pr group was found in other auxiliary-controlled reactions as well [50].

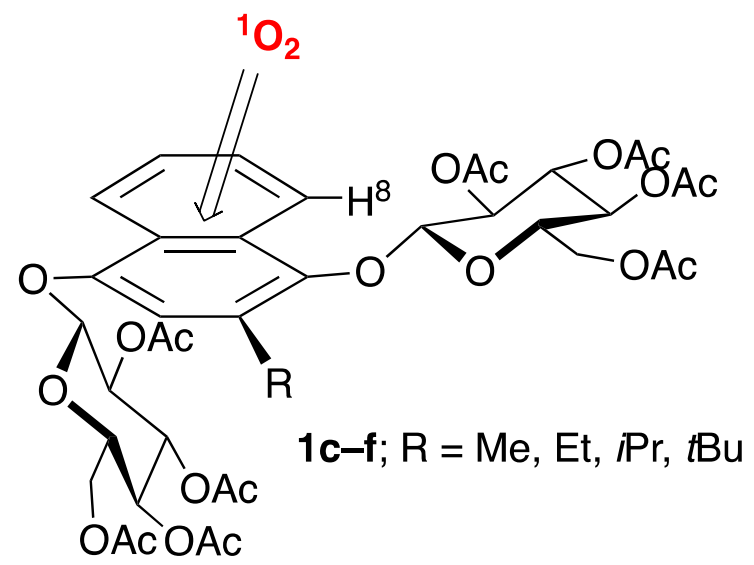

Figure 4. Proposed conformations of naphthalenes $1 \mathbf{c}-\mathbf{f}$ and preferred attack of ${ }^{1} \mathrm{O}_{2}$.

Finally, because our structural assignment was hitherto only based on NOESY measurements and calculations, we wanted to determine the absolute configurations unequivocally. Thus, we selected EPO 1S,4R-2f, which we could isolate in analytically pure form, which may have the same configuration as all main isomers, and which is stable enough for further transformations. Epoxidation with $m$-chloroperoxybenzoic acid (MCPBA) in dichloromethane afforded a single diastereomer syn-7 as shown by the NMR of the crude product. This is in accordance to literature-known epoxidations of simple naphthalene EPOs [51] and can be explained by steric interactions and hydrogen bonding to the peroxide bridge. The epoxide syn-7 was isolated by column chromatography in $76 \%$ yield in analytically pure form (Scheme 3). Finally, the chiral carbohydrate auxiliaries could be removed by catalytic hydrogenation of the $\mathrm{O}-\mathrm{O}$ bond, and naphthoquinone epoxide $2 S, 3 R-8$ was isolated in $63 \%$ yield, besides some decomposition products. Now, we could determine its absolute configuration unequivocally by comparison of the optical rotation with literature-known epoxide $2 R, 3 S-8$, which was synthesized from chiral hydroperoxides in $82 \%$ ee [52]. 


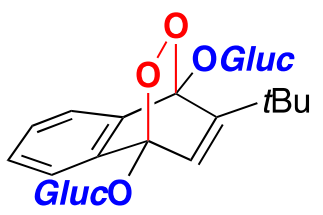

$1 S, 4 R-\mathbf{2 b}-\mathbf{f}$

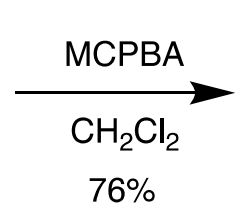

$76 \%$

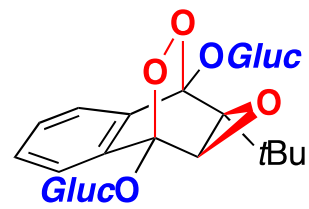

syn-7

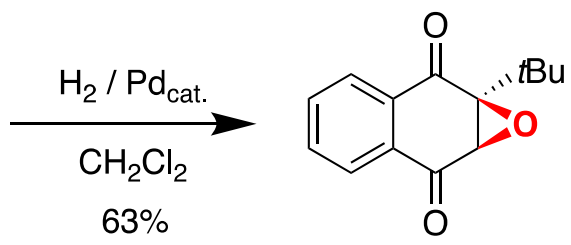

$2 S, 3 R-8(>98 \%$ ee)

Scheme 3. Transformation of EPO $1 S, 4 R-\mathbf{2 f}$ into enantiomerically pure epoxide $2 S, 3 R-\mathbf{8}$.

The fact that our epoxide is enantiomerically pure was confirmed by HPLC on a chiral phase (Figure 5, Section 3). First, we synthesized racemic epoxide 8 by direct oxidation of naphthoquinone $3 \mathbf{f}$ in analogy to literature [53]. This racemate gave two peaks, separated by 2 min retention time (A), and epoxide $2 S, 3 R-8$ gave a single peak (B). Therefore, we could use carbohydrates as chiral auxiliaries to control the photooxygenation of naphthalenes, isolate endoperoxides in pure form, and transfer the chirality to new stereogenic centers under cleavage of the auxiliary, obtaining an enantiomerically pure product.
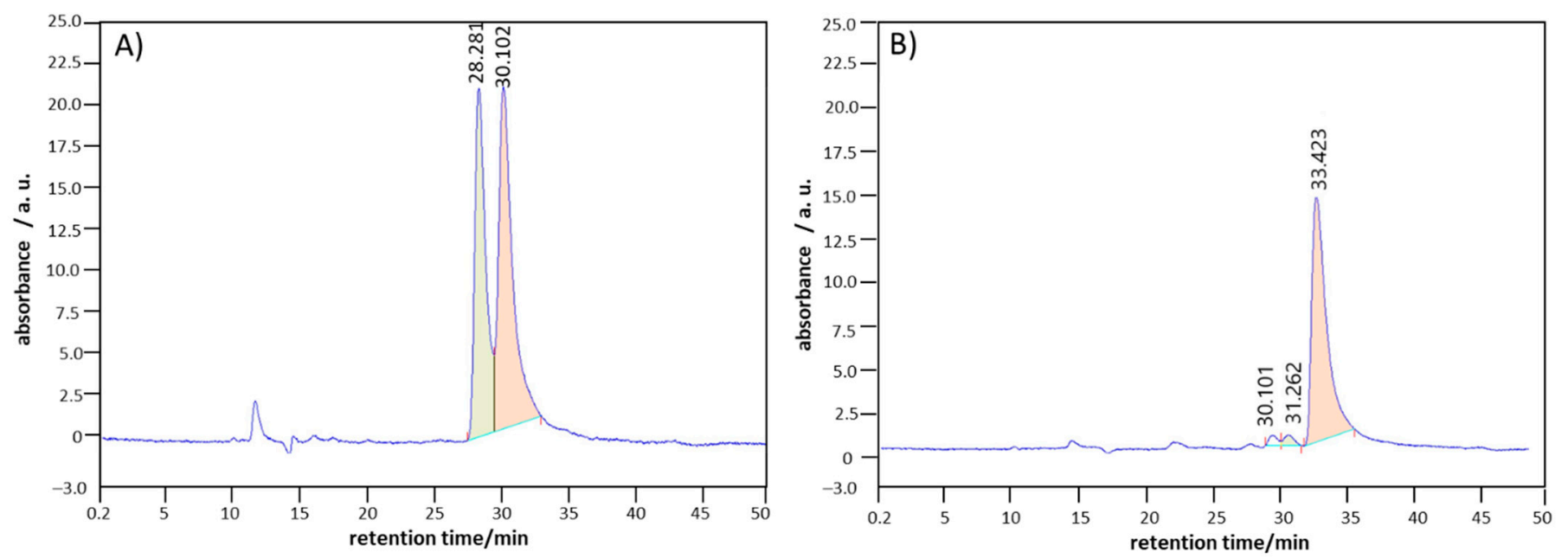

Figure 5. HPLC separation of epoxide $\mathbf{8}$ on chiral phase. (A) racemate $\mathbf{8}$ and (B) epoxide $2 S, 3 R-8.2 R, 3 S$ isomer is marked green; $2 S, 3 R$ isomer is marked red.

\section{Materials and Methods}

\subsection{Chemicals and Instrumentation}

Melting points were determined using a Mel-Temp from Electrothermal. TLC was performed using TLC Silica gel $60 \mathrm{~F} 254$ aluminum sheets from Merck. ${ }^{1} \mathrm{H}-\mathrm{NMR}$ and ${ }^{13} \mathrm{C}$ NMR spectra were measured using a Bruker Avance 300 (300 MHz, $75 \mathrm{MHz}$ ) (Rheinstetten, Germany), a Bruker NEO 500 (500 MHz, $125 \mathrm{MHz}$ ) (Rheinstetten, Germany), or a Bruker Avance 600 (600 MHz, $150 \mathrm{MHz}$ ) NMR spectrometer (Rheinstetten, Germany). The solvents $\left(\mathrm{CDCl}_{3}\right.$ or $\left.\mathrm{CD}_{2} \mathrm{Cl}_{2}\right)$ were used as standard for calibrating chemical shifts. Signals were assigned by two-dimensional methods (HSQC). IR spectra were recorded in $\mathrm{KBr}$ pellets using a Nicolet Avatar 370 FT-IR spectrometer (Madison, WI, USA) from Thermo Electron Corporation. Optical rotations were measured on a JASCO P-1020 digital polarimeter (Tokio, Japan) at $589 \mathrm{~nm}$. Elemental analysis was performed on a Vario EL III elemental analyzer (Elementar, Langenselbold, Germany). HRMS spectra were measured at a GC-MS Trace DSX II spectrometer (Dreieich, Germany). UV spectra were recorded with an Analytik Jena Specord S600 spectrometer (Analytik Jena, Jena, Germany). HPLC was performed with a Chrom Tech, Chiral-AGP column $(100 \mathrm{~mm} \times 3.00 \mathrm{~mm}, 5 \mu \mathrm{m})$, eluent hexane/ethyl acetate 10:1. All starting materials were used as purchased without further purification. Trichloroacetimidate gluco-6 was synthesized according to the literature [54]. 


\subsection{Synthesis of Naphthoquinones 3}

According to the literature [45], 1,4-naphthoquinone $3 \mathbf{b}(3.95 \mathrm{~g}, 25 \mathrm{mmol})$ and the corresponding carboxylic acid 4 (50 mmol, 2 equiv.) were dissolved in a 1:1 mixture of dichloromethane and acetonitrile $(100 \mathrm{~mL})$. This mixture was added to a solution of silver nitrate $(425 \mathrm{mg}, 2.5 \mathrm{mmol})$ and ammonium persulfate $(11.4 \mathrm{~g}, 50 \mathrm{mmol})$ in water $(100 \mathrm{~mL})$ at room temperature. The mixture was heated under reflux for $4 \mathrm{~h}$, cooled to room temperature, and diluted with dichloromethane $(100 \mathrm{~mL})$, and the organic phases were separated. The aqueous phase was extracted with dichloromethane $(3 \times 50 \mathrm{~mL})$, and the combined organic layers were washed with brine $(300 \mathrm{~mL})$ and dried over magnesium sulfate. The solvent was removed in vacuo, and the crude products were purified by column chromatography (PE/EtOAc 10:1) to obtain the naphthoquinones $\mathbf{3 c}-\mathbf{f}$ in analytically pure form.

2-Methyl-1,4-naphthoquinone 3c. Obtained as a yellow solid (1.76 g, 41\%). m.p. $=106-108^{\circ} \mathrm{C}$. $R_{f}=0.23$ (PE/EtOAc 10:1). ${ }^{1} \mathrm{H}-\mathrm{NMR}\left(500 \mathrm{MHz}, \mathrm{CD}_{2} \mathrm{Cl}_{2}\right): \delta=2.16\left(\mathrm{~d}, \mathrm{~J}=1.6 \mathrm{~Hz}, 3 \mathrm{H} ; \mathrm{CH}_{3}\right)$, $6.81(\mathrm{q}, J=1.6 \mathrm{~Hz}, 1 \mathrm{H} ; 3-\mathrm{H}), 6.77(\mathrm{t}, J=1.6 \mathrm{~Hz}, 1 \mathrm{H} ; 3-\mathrm{H}), 7.70-7.74(\mathrm{~m}, 2 \mathrm{H} ; 7-\mathrm{H}, 6-\mathrm{H})$, 7.99-8.02 (m, 1H; H-5), 8.03-8.07 ppm (m, $1 \mathrm{H} ; 8-\mathrm{H}) .{ }^{13} \mathrm{C}-\mathrm{NMR}\left(125 \mathrm{MHz}, \mathrm{CD}_{2} \mathrm{Cl}_{2}\right): \delta=16.5$ (q; $\left.\mathrm{CH}_{3}\right), 126.2(\mathrm{~d} ; \mathrm{C}-5), 126.6(\mathrm{~d} ; \mathrm{C}-8), 132.6(\mathrm{~s} ; \mathrm{C}-8 \mathrm{a}), 132.6(\mathrm{~s} ; \mathrm{C}-4 \mathrm{a}), 133.9(\mathrm{~d} ; \mathrm{C}-7), 134.0(\mathrm{~d} ;$ C-6), 135.9 (d; C-3), 148.5 (s; C-2), 185.2 (s; C-4), 185.8 ppm (s; C-1); IR (Film): v = 3656 (w), $3292(\mathrm{w}), 2956(\mathrm{~m}), 2920(\mathrm{~m}), 1621(\mathrm{~s}), 1588(\mathrm{~m}), 1464(\mathrm{~m}), 1329(\mathrm{~m}), 1300(\mathrm{~s}), 1245(\mathrm{~m})$, $1143(\mathrm{~m}), 1061 \mathrm{~cm}^{-1}$ (m). HRMS (GC-TOF): $\mathrm{m} / z$ Calcd for $\mathrm{C}_{11} \mathrm{H}_{8} \mathrm{O}_{2}\left[\mathrm{M}^{+}\right]: 172.0524$; Found: 172.0533. Anal. Calcd for $\mathrm{C}_{11} \mathrm{H}_{8} \mathrm{O}_{2}$ (172.18): C 76.73, H 4.68; Found: C 77.01, H 4.73.

2-Ethyl-1,4-naphthoquinone 3d. Obtained as a yellow solid (2.35 g, 50\%). m.p. $=83-84{ }^{\circ} \mathrm{C}$. $R_{f}=0.27(\mathrm{PE} / \mathrm{EtOAc} 10: 1) .{ }^{1} \mathrm{H}-\mathrm{NMR}\left(600 \mathrm{MHz}, \mathrm{CD}_{2} \mathrm{Cl}_{2}\right): \delta=1.19\left(\mathrm{t}, J=7.4 \mathrm{~Hz}, 3 \mathrm{H} ; \mathrm{CH}_{3}\right)$, $2.59\left(\mathrm{dq}, J=7.4,1.6 \mathrm{~Hz}, 2 \mathrm{H} ; \mathrm{CH}_{2}\right), 6.77(\mathrm{t}, J=1.6 \mathrm{~Hz}, 1 \mathrm{H} ; 3-\mathrm{H}), 7.72-7.75(\mathrm{~m}, 2 \mathrm{H} ; 7-\mathrm{H}, 6-\mathrm{H})$, 8.01-8.03 (m, 1H; H-5), 8.06-8.09 ppm (m, $1 \mathrm{H} ; 8-\mathrm{H}) .{ }^{13} \mathrm{C}-\mathrm{NMR}\left(150 \mathrm{MHz}, \mathrm{CD}_{2} \mathrm{Cl}_{2}\right): \delta=12.0$ (q; $\left.\mathrm{CH}_{3}\right), 23.0\left(\mathrm{t} ; \mathrm{CH}_{2}\right), 126.2(\mathrm{~d} ; \mathrm{C}-5), 126.7(\mathrm{~d} ; \mathrm{C}-8), 132.5(\mathrm{~s} ; \mathrm{C}-8 \mathrm{a}), 132.8(\mathrm{~s} ; \mathrm{C}-4 \mathrm{a}), 133.9$ (d; C-7), 134.0 (d; C-6), 134.3 (d; C-3), 153.5 (s; C-2), 185.5 (s; C-4), 185.5 ppm (s; C-1). IR (Film): v = $3668(\mathrm{w}), 3312(\mathrm{w}), 2973(\mathrm{~m}), 2906(\mathrm{~m}), 1661(\mathrm{~s}), 1590(\mathrm{~m}), 1458(\mathrm{~m}), 1385(\mathrm{~m})$, $1327(\mathrm{~m}), 1296$ (s), $1250(\mathrm{~m}), 1143(\mathrm{~m}), 1057 \mathrm{~cm}^{-1}(\mathrm{~m})$. HRMS (GC-TOF): $m / z$ Calcd for $\mathrm{C}_{12} \mathrm{H}_{10} \mathrm{O}_{2}\left[\mathrm{M}^{+}\right.$]: 186.0681; Found: 186.0687. Anal. Calcd for $\mathrm{C}_{12} \mathrm{H}_{10} \mathrm{O}_{2}$ (186.21): C 77.40, H 5.41; Found: C 77.40, H 5.71.

2-iPropyl-1,4-naphthoquinone 3e. Obtained as a yellow solid (3.21 g, 64\%). m.p. $=43-44{ }^{\circ} \mathrm{C}$. $R_{f}=0.34(\mathrm{PE} / \mathrm{EtOAc} 10: 1) .{ }^{1} \mathrm{H}-\mathrm{NMR}\left(600 \mathrm{MHz}, \mathrm{CD}_{2} \mathrm{Cl}_{2}\right): \delta=1.19\left(2 \mathrm{~d}, J=6.9 \mathrm{~Hz}, 6 \mathrm{H} ; \mathrm{CH}_{3}\right)$, 3.22 (dsept, $J=6.9,1.2 \mathrm{~Hz}, 1 \mathrm{H} ; \mathrm{CH}), 6.76(\mathrm{~d}, J=1.2 \mathrm{~Hz}, 1 \mathrm{H} ; 3-\mathrm{H}), 7.72-7.76(\mathrm{~m}, 2 \mathrm{H} ; 6-\mathrm{H}$, 7-H), 8.01-8.04 (m, 1H; H-5), 8.07-8.10 ppm (m, 1H; 8-H). ${ }^{13} \mathrm{C}-\mathrm{NMR}\left(150 \mathrm{MHz}, \mathrm{CD}_{2} \mathrm{Cl}_{2}\right)$ : $\delta=21.7\left(2 \mathrm{q} ; \mathrm{CH}_{3}\right), 27.4(\mathrm{~d} ; \mathrm{CH}), 126.1(\mathrm{~d} ; \mathrm{C}-5), 126.8(\mathrm{~d} ; \mathrm{C}-8), 132.3(\mathrm{~s} ; \mathrm{C}-4 \mathrm{a}), 133.0(\mathrm{~s} ; \mathrm{C}-8 \mathrm{a})$, 133.0 (d; C-3), 133.9 (d; C-7), 134.0 (d; C-6), 157.5 (s; C-2), 185.1 (s; C-1), 185.8 ppm (s; C-4). IR (Film): v = 3660 (w), 3315 (w), 3071 (w), 2967 (m), $2873(\mathrm{~m}), 1660$ (s), $1594(\mathrm{~m}), 1463(\mathrm{w})$, $1379(\mathrm{w}), 1328(\mathrm{~m}), 1299$ (s), 1251 (s), 1130 (w), $1074 \mathrm{~cm}^{-1}$ (m). HRMS (GC-TOF): $m / z$ Calcd for $\mathrm{C}_{13} \mathrm{H}_{12} \mathrm{O}_{2}$ [M+]: 200.0837; Found: 200.0832. Anal. Calcd for $\mathrm{C}_{13} \mathrm{H}_{12} \mathrm{O}_{2}$ (200.23): C 77.98, H 6.04; Found: C 77.84, H 5.94.

2-tButyl-1,4-naphthoquinone 3f. Obtained as a yellow solid (3.89 g, 73\%). m.p. $=66-67^{\circ} \mathrm{C}$. $R_{f}=0.42(\mathrm{PE} / \mathrm{EtOAc} 10: 1) .{ }^{1} \mathrm{H}-\mathrm{NMR}\left(600 \mathrm{MHz}, \mathrm{CD}_{2} \mathrm{Cl}_{2}\right): \delta=1.36(\mathrm{~s}, 9 \mathrm{H} ; \mathrm{t}-\mathrm{Bu}), 6.83(\mathrm{~s}, 1 \mathrm{H}$; 3-H), $7.71(\mathrm{ddd}, J=8.9,7.4,1.5 \mathrm{~Hz}, 1 \mathrm{H} ; 6-\mathrm{H}), 7.74(\mathrm{ddd}, J=8.9,7.4,1.6 \mathrm{~Hz}, 1 \mathrm{H} ; \mathrm{H}-7), 8.00$ (ddd, $J=7.4,1.6,0.6 \mathrm{~Hz}, 1 \mathrm{H} ; \mathrm{H}-5), 8.07 \mathrm{ppm}(\mathrm{ddd}, J=7.4,1.5,0.6 \mathrm{~Hz}, 1 \mathrm{H} ; 8-\mathrm{H}) .{ }^{13} \mathrm{C}-\mathrm{NMR}$ $\left(150 \mathrm{MHz}, \mathrm{CD}_{2} \mathrm{Cl}_{2}\right): \delta=29.5(\mathrm{q} ; \mathrm{t}-\mathrm{Bu}), 36.0(\mathrm{~s} ; \mathrm{t}-\mathrm{Bu}), 125.7$ (d; C-5), $127.0(\mathrm{~d} ; \mathrm{C}-8), 131.9(\mathrm{~s} ;$ C-4a), 133.6 (d; C-6), 134.0 (s; C-8a), 134.1 (d; C-7), 134.3 (d; C-3), 158.5 (s; C-2), 185.3 (s; C-1), 186.1 ppm (s; C-4). IR (Film): v = 3306 (w), 3071 (w), 2963 (m), $2910(\mathrm{~m}), 2874(\mathrm{~m})$, $1792(\mathrm{w}), 1655(\mathrm{~s}), 1594(\mathrm{~s}), 1485(\mathrm{~m}), 1460(\mathrm{~m}), 1362(\mathrm{~m}), 1331(\mathrm{~s}), 1308(\mathrm{~s}), 1249(\mathrm{~s}), 1202(\mathrm{~m})$, $1127 \mathrm{~cm}^{-1}$ (s). HRMS (GC-TOF): $m / z$ Calcd for $\mathrm{C}_{14} \mathrm{H}_{14} \mathrm{O}_{2}\left[\mathrm{M}^{+}\right]$: 214.0994; Found: 214.0988. Anal. Calcd for $\mathrm{C}_{14} \mathrm{H}_{14} \mathrm{O}_{2}$ (214.26): C 78.48, H 6.59; Found: C 78.56, H 6.68. 


\subsection{Synthesis of Carbohydrate-Substituted Naphthalenes $\mathbf{1}$}

In short, 1,4-Naphthoquinone 3 (5 mmol) was dissolved in EtOAc $(15 \mathrm{~mL})$ and an aqueous solution of sodium dithionite $(2.61 \mathrm{~g}, 15 \mathrm{mmol}, 15 \mathrm{~mL})$ was added under nitrogen atmosphere at room temperature. After stirring for $3 \mathrm{~h}$, the mixture was diluted with a saturated solution of ammonium chloride $(15 \mathrm{~mL})$ and extracted with EtOAc $(3 \times 25 \mathrm{~mL})$. The combined organic layers were washed with water $(50 \mathrm{~mL})$ and brine $(50 \mathrm{~mL})$ and dried over magnesium sulfate. The solvent was removed in vacuo, and the crude 1,4dihydroxynaphthalene 5 was directly used for the next step.

The crude 1,4-dihydroxynapthalene 5 was dissolved in dry dichloromethane $(50 \mathrm{~mL})$, molecular sieves $(4 \AA)$, and a solution of trichloroacetimidate $6(4.93 \mathrm{~g}, 10 \mathrm{mmol})$ in dry dichloromethane $(50 \mathrm{~mL})$ was added at room temperature. The mixture was cooled to $-15^{\circ} \mathrm{C}$, and a solution of $\mathrm{BF}_{3} \cdot \mathrm{Et}_{2} \mathrm{O}(0.13 \mathrm{~mL}, 1 \mathrm{mmol})$ in dry dichloromethane $(5 \mathrm{~mL})$ was added within $30 \mathrm{~min}$. After stirring for $16 \mathrm{~h}$ at room temperature, the mixture was filtered over celite; washed with saturated solution of $\mathrm{NaHCO}_{3}(50 \mathrm{~mL})$, water $(50 \mathrm{~mL})$, and brine $(50 \mathrm{~mL})$; and dried over magnesium sulfate. The solvent was removed in vacuo, and the crude products were purified by column chromatography (hexanes/EtOAc 1:1) to obtain the carbohydrate-substituted naphthalenes $\mathbf{1}$ in analytically pure form.

1,4-Bis-(1-O-2,3,4,6-tetra-O-acetyl- $\beta$-D-glucopyranosyl)-naphthalene $\mathbf{1 b}$. Obtained as a colorless solid $\left(2.923\right.$ g, $76 \%$ ). m.p. $=169-171{ }^{\circ} \mathrm{C} . R_{f}=0.18$ (PE/EtOAc 1:1). $[\alpha]_{D}^{24}=-67$ (c 1.00, $\left.\mathrm{CHCl}_{3}\right) .{ }^{1} \mathrm{H}-\mathrm{NMR}\left(600 \mathrm{MHz}, \mathrm{CD}_{2} \mathrm{Cl}_{2}\right.$ ): $\delta=2.04$ (2s, 6H; 3-OAc), 2.04 (2s, 6H; 4-OAc), 2.05 (2s, 6H; 2-OAc), 2.06 (2s, 6H; 6-OAc), 3.94 (2ddd, $J=10.0,5.0,2.5 \mathrm{~Hz}, 2 \mathrm{H} ; 5-\mathrm{H}), 4.20$ (2dd, $J=12.3,2.5 \mathrm{~Hz}, 2 \mathrm{H} ; 6 \mathrm{a}-\mathrm{H}), 4.32(2 \mathrm{dd}, J=12.3,5.0 \mathrm{~Hz}, 2 \mathrm{H} ; 6 \mathrm{~b}-\mathrm{H}), 5.21(2 \mathrm{dd}, J=10.0,9.4 \mathrm{~Hz}$, $2 \mathrm{H} ; 4-\mathrm{H}), 5.21(2 \mathrm{~d}, J=7.9 \mathrm{~Hz}, 2 \mathrm{H} ; 1-\mathrm{H}), 5.36(2 \mathrm{dd}, J=9.7,9.4 \mathrm{~Hz}, 2 \mathrm{H} ; 3-\mathrm{H}), 5.43(2 \mathrm{dd}$, $J=9.7,7.9 \mathrm{~Hz}, 2 \mathrm{H} ; 2-\mathrm{H}), 7.02$ (2s, 2H; 2-ArH, 3-ArH), 7.53-7.56 (m, 2H; 6-ArH, 7-ArH), 8.02-8.05 ppm (m, 2H; 5-ArH, 8-ArH). ${ }^{13} \mathrm{C}-\mathrm{NMR}\left(150 \mathrm{MHz}, \mathrm{CD}_{2} \mathrm{Cl}_{2}\right): \delta=20.8$ (4q; OAc-2, OAc-3), 20.9 (2q; OAc-6), 20.9 (2q; OAc-4), 62.2 (2t; C-6), 68.7 (2d; C-4), 71.4 (2d; C-2), 72.5 (2d; C-5), 72.8 (2d; C-3), 100.3 (2d; C-1), 109.7 (2d; ArC-2, ArC-3), 121.8 (2d; ArC-5, ArC-8), 126.9 (2s; ArC-4a, ArC-8a), 127.1 (2d; ArC-6, ArC-7), 149.0 (2s; ArC-1, ArC-4), 169.8 (2s; OAc-4), 169.9 (2s; OAc-2), 170.3 (2s; OAc-3), 170.7 ppm (2s; OAc-6). IR (Film): $v=3024(\mathrm{w}), 2960(\mathrm{w}), 1746(\mathrm{~s}), 1599(\mathrm{w}), 1371(\mathrm{~m}), 1212(\mathrm{~s}), 1122(\mathrm{~m}), 1035 \mathrm{~cm}^{-1}(\mathrm{~s})$. HRMS (ESI-Q-TOF): $m / z$ Calcd for $\mathrm{C}_{38} \mathrm{H}_{44} \mathrm{NaO}_{20}\left[\mathrm{M}+\mathrm{Na}^{+}\right.$]: 843.2324; Found: 843.2296. Anal. Calcd for $\mathrm{C}_{38} \mathrm{H}_{44} \mathrm{O}_{20}$ (820.75): C 55.61, H 5.40; Found: C 55.61, H 5.55.

2-Methyl-1,4-bis-(1-O-2,3,4,6-tetra-O-acetyl- $\beta$-D-glucopyranosyl)-naphthalene 1c. Obtained as a colorless solid $(2.994 \mathrm{~g}, 72 \%)$. m.p. $=216-217^{\circ} \mathrm{C} . R_{f}=0.17$ (PE/EtOAc 1:1). $[\alpha]_{D}^{24}=-34(c$ 1.00, $\mathrm{CHCl}_{3}$ ). ${ }^{1} \mathrm{H}-\mathrm{NMR}\left(600 \mathrm{MHz}, \mathrm{CD}_{2} \mathrm{Cl}_{2}\right.$ ): $\delta=1.88$ (s, 3H; 6-OAc), 1.99 (s, 3H; 4-OAc), 2.03 (s, 3H; 2'-OAc), 2.03 (s, 3H; 3-OAc), 2.04 (s, 3H; 3'-OAc), 2.05 (s, 3H; 4'-OAc), 2.08 (s, 3H; 6'-OAc), 2.18 (s, 3H; 2-OAc), $2.46\left(\mathrm{~s}, 3 \mathrm{H} ; \mathrm{CH}_{3}\right), 3.56$ (ddd, $J=10.0,5.1,2.6 \mathrm{~Hz}, 1 \mathrm{H}$; $5-\mathrm{H}), 3.98(\mathrm{dd}, J=12.2,2.6 \mathrm{~Hz}, 1 \mathrm{H} ; 6 \mathrm{a}-\mathrm{H}), 4.01\left(\mathrm{ddd}, J=10.1,5.8,2.5 \mathrm{~Hz}, 1 \mathrm{H}, 5^{\prime}-\mathrm{H}\right), 4.17$ $(\mathrm{dd}, J=12.2,5.1 \mathrm{~Hz}, 1 \mathrm{H} ; 6 \mathrm{~b}-\mathrm{H}), 4.22\left(\mathrm{dd}, J=12.3,2.5 \mathrm{~Hz}, 1 \mathrm{H} ; 6 \mathrm{a}^{\prime}-\mathrm{H}\right), 4.28(\mathrm{dd}, J=12.3$, $\left.5.8 \mathrm{~Hz}, 1 \mathrm{H} ; 6 \mathrm{~b}^{\prime}-\mathrm{H}\right), 5.05(\mathrm{~d}, J=8.1 \mathrm{~Hz}, 1 \mathrm{H} ; 1-\mathrm{H}), 5.18(\mathrm{dd}, J=10.0,9.4 \mathrm{~Hz}, 1 \mathrm{H} ; 4-\mathrm{H}), 5.18$ $\left(\mathrm{dd}, J=10.1,9.3 \mathrm{~Hz}, 1 \mathrm{H} ; 4^{\prime}-\mathrm{H}\right), 5.26\left(\mathrm{~d}, J=7.8 \mathrm{~Hz}, 1 \mathrm{H} ; 1^{\prime}-\mathrm{H}\right), 5.30(\mathrm{dd}, J=9.8,9.4 \mathrm{~Hz}, 1 \mathrm{H}$; 3-H), $5.38\left(\mathrm{dd}, J=9.8,9.3 \mathrm{~Hz}, 1 \mathrm{H} ; 3^{\prime}-\mathrm{H}\right), 5.44(\mathrm{dd}, J=9.8,8.1 \mathrm{~Hz}, 1 \mathrm{H} ; 2-\mathrm{H}), 5.45(\mathrm{dd}, J=9.8$, $\left.7.8 \mathrm{~Hz}, 1 \mathrm{H} ; 2^{\prime}-\mathrm{H}\right), 6.94(\mathrm{~s}, 1 \mathrm{H} ; 3-\mathrm{ArH}), 7.44(\mathrm{ddd}, J=8.4,6.8,1.2 \mathrm{~Hz}, 1 \mathrm{H} ; 6-\mathrm{ArH}), 7.52$ (ddd, $J=8.6,6.8,1.3 \mathrm{~Hz}, 1 \mathrm{H} ; 7-\mathrm{ArH}), 8.00$ (ddd, $J=8.4,1.3,0.6 \mathrm{~Hz}, 1 \mathrm{H} ; 5-\mathrm{ArH}), 8.12 \mathrm{ppm}$ (ddd, $J=8.6,1.2,0.6 \mathrm{~Hz}, 1 \mathrm{H} ; 8-\mathrm{ArH}) .{ }^{13} \mathrm{C}-\mathrm{NMR}\left(150 \mathrm{MHz}, \mathrm{CD}_{2} \mathrm{Cl}_{2}\right): \delta=17.5\left(\mathrm{q} ; \mathrm{CH}_{3}\right), 20.6(\mathrm{q} ;$ OAc-6), 20.7 (q; OAc-4), 20.8 (q; OAc-2') $20.8\left(2 \mathrm{q} ; \mathrm{OAc}^{\prime}-2, \mathrm{OAc}^{\prime} 3^{\prime}\right), 20.9$ (2q; OAc-4', OAc-6'), $21.0\left(\mathrm{q} ;\right.$ OAc-2), $62.0(\mathrm{t} ; \mathrm{C}-6), 62.6\left(\mathrm{t} ; \mathrm{C}-6^{\prime}\right), 68.8(\mathrm{~d} ; \mathrm{C}-4), 68.9\left(\mathrm{~d} ; \mathrm{C}-4^{\prime}\right), 71.4\left(\mathrm{~d} ; \mathrm{C}-2^{\prime}\right), 72.1$ (C-5), $72.2(\mathrm{~d} ; \mathrm{C}-2), 72.5\left(\mathrm{~d} ; \mathrm{C}-5^{\prime}\right), 72.7\left(\mathrm{~d} ; \mathrm{C}-3^{\prime}\right), 73.1(\mathrm{~d} ; \mathrm{C}-3), 99.9\left(\mathrm{~d} ; \mathrm{C}-1^{\prime}\right), 102.4(\mathrm{~d} ; \mathrm{C}-1)$, 112.6 (d; ArC-3), 121.9 (d; ArC-5), 122.2 (d; ArC-8), 125.5 (s; ArC-4a), 125.8 (s; ArC-6), 127.1 (d; ArC-7), 128.2 (s; ArC-8a), 129.0 (s; ArC-2), 144.8 (s; ArC-1), 150.0 (s; ArC-4), 169.6 (s; OAc-2), 169.8 (s; OAc-2'), 169.8 (s; OAc-4), 169.8 (s; OAc-4), 170.3 (s; OAc-3'), 170.4 (s; OAc-3), 170.5 (s; OAc-6), 170.7 ppm (s; OAc-6'). IR (Film): v = 2968 (m), 2899 (w), $1742(\mathrm{~s})$, $1367(\mathrm{~m}), 1220(\mathrm{~s}), 1170(\mathrm{~m}), 1123(\mathrm{~m}), 1038 \mathrm{~cm}^{-1}$ (s). HRMS (ESI-Q-TOF): $m / z$ Calcd for 
$\mathrm{C}_{39} \mathrm{H}_{46} \mathrm{NaO}_{20}\left[\mathrm{M}+\mathrm{Na}^{+}\right]$: 857.2475; Found: 857.2477. Anal. Calcd for $\mathrm{C}_{39} \mathrm{H}_{46} \mathrm{O}_{20}$ (834.78): C 56.11, H 5.55; Found: C 56.03, H 5.61.

2-Ethyl-1,4-bis-(1-O-2,3,4,6-tetra-O-acetyl- $\beta$-D-glucopyranosyl)-naphthalene 1d. Obtained as a colorless solid (3.155 g, 74\%). m.p. $=218-219^{\circ} \mathrm{C} . R_{f}=0.22\left(\right.$ PE $/$ EtOAc 1:1). $[\alpha]_{D}^{23}=-28(c$ $\left.1.01, \mathrm{CHCl}_{3}\right) .{ }^{1} \mathrm{H}-\mathrm{NMR}\left(600 \mathrm{MHz}, \mathrm{CD}_{2} \mathrm{Cl}_{2}\right): \delta=1.25\left(\mathrm{t}, J=7.6 \mathrm{~Hz}, 3 \mathrm{H} ; \mathrm{CH}_{3}\right), 1.89(\mathrm{~s}, 3 \mathrm{H} ;$ 6-OAc), 1.99 (s, 3H; 4-OAc), 2.03 (s, 3H; 2'-OAc), 2.03 (s, 3H; 3-OAc), 2.04 (s, 3H; 3'-OAc), 2.05 (s, 3H; 4'-OAc), 2.07 (s, 3H; 6'-OAc), 2.19 (s, 3H; 2-OAc), $2.88\left(\mathrm{q}, J=7.6 \mathrm{~Hz}, 2 \mathrm{H} ; \mathrm{CH}_{2}\right)$, 3.55 (ddd, $J=10.0,5.0,2.6 \mathrm{~Hz}, 1 \mathrm{H} ; 5-\mathrm{H}), 3.94(\mathrm{dd}, J=12.2,2.6 \mathrm{~Hz}, 1 \mathrm{H} ; 6 \mathrm{a}-\mathrm{H}), 4.00$ (ddd, $\left.J=10.1,5.7,2.5 \mathrm{~Hz}, 1 \mathrm{H}, 5^{\prime}-\mathrm{H}\right), 4.15(\mathrm{dd}, J=12.2,5.0 \mathrm{~Hz}, 1 \mathrm{H} ; 6 \mathrm{~b}-\mathrm{H}), 4.22(\mathrm{dd}, J=12.3,2.5 \mathrm{~Hz}$, $\left.1 \mathrm{H} ; 6 \mathrm{a}^{\prime}-\mathrm{H}\right), 4.26\left(\mathrm{dd}, J=12.3,5.7 \mathrm{~Hz}, 1 \mathrm{H} ; 6 \mathrm{~b}^{\prime}-\mathrm{H}\right), 5.04(\mathrm{~d}, J=8.1 \mathrm{~Hz}, 1 \mathrm{H} ; 1-\mathrm{H}), 5.17(\mathrm{dd}$, $J=10.0,9.4 \mathrm{~Hz}, 1 \mathrm{H} ; 4-\mathrm{H}), 5.18\left(\mathrm{dd}, J=10.1,9.3 \mathrm{~Hz}, 1 \mathrm{H} ; 4^{\prime}-\mathrm{H}\right), 5.26\left(\mathrm{~d}, J=7.8 \mathrm{~Hz}, 1 \mathrm{H} ; 1^{\prime}-\mathrm{H}\right)$, $5.29(\mathrm{dd}, J=9.8,9.4 \mathrm{~Hz}, 1 \mathrm{H} ; 3-\mathrm{H}), 5.37\left(\mathrm{dd}, J=9.8,9.3 \mathrm{~Hz}, 1 \mathrm{H} ; 3^{\prime}-\mathrm{H}\right), 5.44(\mathrm{dd}, J=9.8,8.1 \mathrm{~Hz}$, $1 \mathrm{H} ; 2-\mathrm{H}), 5.44\left(\mathrm{dd}, J=9.8,7.8 \mathrm{~Hz}, 1 \mathrm{H} ; 2^{\prime}-\mathrm{H}\right), 6.98(\mathrm{~s}, 1 \mathrm{H} ; 3-\mathrm{ArH}), 7.46(\mathrm{ddd}, J=8.4,6.8$, $1.3 \mathrm{~Hz}, 1 \mathrm{H} ; 6-\mathrm{ArH}), 7.52$ (ddd, $J=8.5,6.8,1.4 \mathrm{~Hz}, 1 \mathrm{H} ; 7-\mathrm{ArH}), 8.00$ (ddd, $J=8.4,1.4,0.6 \mathrm{~Hz}$, $1 \mathrm{H} ; 5-\mathrm{ArH}), 8.14 \mathrm{ppm}(\mathrm{ddd}, J=8.5,1.3,0.6 \mathrm{~Hz}, 1 \mathrm{H} ; 8-\mathrm{ArH}) .{ }^{13} \mathrm{C}-\mathrm{NMR}\left(150 \mathrm{MHz}, \mathrm{CD}_{2} \mathrm{Cl}_{2}\right)$ : $\delta=15.1\left(\mathrm{q} ; \mathrm{CH}_{3}\right), 20.7(\mathrm{q} ; \mathrm{OAc}-6), 20.8(\mathrm{q} ; \mathrm{OAc}-4), 20.8\left(\mathrm{q} ; \mathrm{OAc}-2^{\prime}\right), 20.8\left(2 \mathrm{q} ; \mathrm{OAc}-2, \mathrm{OAc}-3^{\prime}\right)$, $20.8\left(\mathrm{q} ; \mathrm{OAc}^{\prime} 4^{\prime}\right), 20.9\left(\mathrm{q} ; \mathrm{OAc}^{\prime}\right), 21.0(\mathrm{q} ; \mathrm{OAc}-2), 23.5\left(\mathrm{t} ; \mathrm{CH}_{2}\right), 62.0(\mathrm{t} ; \mathrm{C}-6), 62.6\left(\mathrm{t} ; \mathrm{C}-6^{\prime}\right)$, $68.8(\mathrm{~d} ; \mathrm{C}-4), 68.8\left(\mathrm{~d} ; \mathrm{C}-4^{\prime}\right), 71.4\left(\mathrm{~d} ; \mathrm{C}-2^{\prime}\right), 72.2(\mathrm{C}-5), 72.2(\mathrm{~d} ; \mathrm{C}-2), 72.7\left(\mathrm{~d} ; \mathrm{C}-5^{\prime}\right), 72.7(\mathrm{~d}$; C-3'), 73.2 (d; C-3), 100.0 (d; C-1'), 102.4 (d; C-1), 110.9 (d; ArC-3), 121.9 (d; ArC-5), 122.5 (d; ArC-8), 125.6 (s; ArC-4a), 125.9 (s; ArC-6), 127.1 (d; ArC-7), 129.0 (s; ArC-8a), 134.2 (s; ArC-2), 144.0 (s; ArC-1), 150.4 (s; ArC-4), 169.6 (s; OAc-2'), 169.8 (s; OAc-2), 169.8 (s; OAc-4'), 169.8 (s; OAc-4), 170.3 (s; OAc-3'), 170.4 (s; OAc-3), 170.6 (s; OAc-6), 170.8 ppm (s; OAc-6'). IR (Film): v = 3667 (w), 3482 (w), 2970 (m), 2901 (m), 1746 (s), 1365 (m), 1211 (s), 1170 (m), $1034 \mathrm{~cm}^{-1}$ (s). HRMS (ESI-Q-TOF): $m / z$ Calcd for $\mathrm{C}_{40} \mathrm{H}_{48} \mathrm{NaO}_{20}\left[\mathrm{M}+\mathrm{Na}^{+}\right]$: 871.2631; Found: 871.2634. Anal. Calcd for $\mathrm{C}_{40} \mathrm{H}_{48} \mathrm{O}_{20}$ (848.80): C 56.60, H 5.70; Found: C 56.85, H 5.83 .

2-iPropyl-1,4-bis-(1-O-2,3,4,6-tetra-O-acetyl- $\beta$-D-glucopyranosyl)-naphthalene 1e. Obtained as a colorless solid (3.375 g, 74\%). m.p. $=219-220{ }^{\circ} \mathrm{C} . R_{f}=0.27\left(\right.$ PE $/$ EtOAc 1:1). $[\alpha]_{D}^{23}=-26$ (c 1.01, $\left.\mathrm{CHCl}_{3}\right) .{ }^{1} \mathrm{H}-\mathrm{NMR}\left(600 \mathrm{MHz}, \mathrm{CD}_{2} \mathrm{Cl}_{2}\right): \delta=1.23\left(\mathrm{~d}, J=6.9 \mathrm{~Hz}, 3 \mathrm{H} ; \mathrm{CH}_{3}\right), 1.24(\mathrm{~d}$, $\left.J=6.9 \mathrm{~Hz}, 3 \mathrm{H} ; \mathrm{CH}_{3}\right), 1.91$ (s, 3H; 6-OAc), 1.99 (s, 3H; 4-OAc), 2.03 (s, 3H; 3-OAc), 2.04 (2s, 6H; 2'-OAc, 3'-OAc), 2.05 (s, 3H; 4'-OAc), 2.06 (s, 3H; 6'-OAc), 2.20 (s, 3H; 2-OAc), 3.55 (ddd, $J=10.0,4.8,2.5 \mathrm{~Hz}, 1 \mathrm{H} ; 5-\mathrm{H}), 2.88$ (sept; $J=6.9 \mathrm{~Hz}, 1 \mathrm{H} ; \mathrm{CH}), 3.95$ (dd, $J=12.3$, $2.5 \mathrm{~Hz}, 1 \mathrm{H} ; 6 \mathrm{a}-\mathrm{H}), 4.01\left(\mathrm{ddd}, J=10.1,5.2,2.8 \mathrm{~Hz}, 1 \mathrm{H}, 5^{\prime}-\mathrm{H}\right), 4.13(\mathrm{dd}, J=12.3,4.8 \mathrm{~Hz}, 1 \mathrm{H}$; $6 \mathrm{~b}-\mathrm{H}), 4.22\left(\mathrm{dd}, J=12.4,2.8 \mathrm{~Hz}, 1 \mathrm{H} ; 6 \mathrm{a}^{\prime}-\mathrm{H}\right), 4.25\left(\mathrm{dd}, J=12.4,5.2 \mathrm{~Hz}, 1 \mathrm{H} ; 6 \mathrm{~b}^{\prime}-\mathrm{H}\right), 5.05(\mathrm{~d}$, $J=8.0 \mathrm{~Hz}, 1 \mathrm{H} ; 1-\mathrm{H}), 5.17(\mathrm{dd}, J=10.0,9.4 \mathrm{~Hz}, 1 \mathrm{H} ; 4-\mathrm{H}), 5.18\left(\mathrm{dd}, J=10.1,9.3 \mathrm{~Hz}, 1 \mathrm{H} ; 4^{\prime}-\mathrm{H}\right)$, $5.27\left(\mathrm{~d}, J=7.8 \mathrm{~Hz}, 1 \mathrm{H} ; 1^{\prime}-\mathrm{H}\right), 5.30(\mathrm{dd}, J=9.8,9.4 \mathrm{~Hz}, 1 \mathrm{H} ; 3-\mathrm{H}), 5.38(\mathrm{dd}, J=9.7,9.3 \mathrm{~Hz}$, $\left.1 \mathrm{H} ; 3^{\prime}-\mathrm{H}\right), 5.44\left(\mathrm{dd}, J=9.7,7.8 \mathrm{~Hz}, 1 \mathrm{H} ; 2^{\prime}-\mathrm{H}\right), 5.44(\mathrm{dd}, J=9.8,8.0 \mathrm{~Hz}, 1 \mathrm{H} ; 2-\mathrm{H}), 7.02(\mathrm{~s}$, $1 \mathrm{H}$; 3-ArH), 7.47 (ddd, $J=8.4,6.8,1.2 \mathrm{~Hz}, 1 \mathrm{H} ; 6-\mathrm{ArH}), 7.53(\mathrm{ddd}, J=8.5,6.8,1.3 \mathrm{~Hz}, 1 \mathrm{H}$; 7-ArH), 8.01 (ddd, $J=8.4,1.3,0.7 \mathrm{~Hz}, 1 \mathrm{H} ; 5-\mathrm{ArH}), 8.14 \mathrm{ppm}(\mathrm{ddd}, J=8.5,1.2,0.7 \mathrm{~Hz}, 1 \mathrm{H}$; 8-ArH). ${ }^{13} \mathrm{C}-\mathrm{NMR}\left(150 \mathrm{MHz}, \mathrm{CD}_{2} \mathrm{Cl}_{2}\right.$ ): $\delta=20.7$ (q;OAc-6), 20.8 (q; OAc-4), 20.8 (q; OAc-2), $20.8\left(2 \mathrm{q} ; \mathrm{OAc}^{\prime}{ }^{\prime}, \mathrm{OAc}^{\prime} 3^{\prime}\right), 20.9$ (q; OAc- $\left.4^{\prime}\right), 21.0\left(\mathrm{q} ; \mathrm{OAc}-6^{\prime}\right), 21.0(\mathrm{q} ; \mathrm{OAc}-2), 23.4\left(\mathrm{q} ; \mathrm{CH}_{3}\right)$, $23.9\left(\mathrm{q} ; \mathrm{CH}_{3}\right), 26.6(\mathrm{~d} ; \mathrm{CH}), 62.1(\mathrm{t} ; \mathrm{C}-6), 62.7\left(\mathrm{t} ; \mathrm{C}-6^{\prime}\right), 68.7(\mathrm{~d} ; \mathrm{C}-4), 68.7\left(\mathrm{~d} ; \mathrm{C}-4^{\prime}\right), 71.3(\mathrm{~d} ;$ C-2'), 72.2 (C-5), 72.2 (d; C-2), $72.7\left(\mathrm{~d} ; \mathrm{C}-3^{\prime}\right), 72.7$ (d; C-5'), 73.2 (d; C-3), 100.0 (d; C-1'), 102.3 (d; C-1), 108.0 (d; ArC-3), 121.9 (d; ArC-5), 122.4 (d; ArC-8), 125.6 (s; ArC-4a), 125.9 (s; ArC-6), 127.1 (d; ArC-7), 128.8 (s; ArC-8a), 138.9 (s; ArC-2), 142.8 (s; ArC-1), 150.7 (s; ArC-4), $169.6(\mathrm{~s} ;$ OAc-2'), 169.7 (s; OAc-2), $169.8(\mathrm{~s} ;$ OAc-4), $169.8(\mathrm{~s} ;$ OAc-4'), $170.3(\mathrm{~s} ;$ OAc-3'), 170.5 (s; OAc-3), 170.6 (s; OAc-6), 170.8 ppm (s; OAc-6' ${ }^{\prime}$. IR (Film): v = $3667(\mathrm{w}), 3482(\mathrm{w})$, $2969(\mathrm{~m}), 2897(\mathrm{w}), 1746(\mathrm{~s}), 1601(\mathrm{w}), 1365(\mathrm{~m}), 1212(\mathrm{~s}), 1171(\mathrm{~m}), 1035 \mathrm{~cm}^{-1}(\mathrm{~s})$. HRMS (ESI-Q-TOF): $m / z$ Calcd for $\mathrm{C}_{41} \mathrm{H}_{50} \mathrm{NaO}_{20}\left[\mathrm{M}+\mathrm{Na}^{+}\right]$: 871.2631; Found: 871.2634. Anal. Calcd for $\mathrm{C}_{41} \mathrm{H}_{50} \mathrm{O}_{20}$ (862.83): C 57.07, H 5.84; Found: C 56.98, H 5.90.

2-tButyl-1,4-bis-(1-O-2,3,4,6-tetra-O-acetyl- $\beta$-D-glucopyranosyl)-naphthalene 1f. Obtained as a colorless solid (3.106 g, 71\%). m.p. $=230-231{ }^{\circ} \mathrm{C} . R_{f}=0.31(\mathrm{PE} /$ EtOAc $1: 1) \cdot[\alpha]_{D}^{24}=-28$ (c 1.01, $\left.\mathrm{CHCl}_{3}\right) .{ }^{1} \mathrm{H}-\mathrm{NMR}\left(600 \mathrm{MHz}, \mathrm{CD}_{2} \mathrm{Cl}_{2}\right): \delta=1.49$ (s, 9H; t-Bu), 1.93 (s, 3H; 6-OAc), 
1.98 (s, 3H; 4'-OAc), 2.03 (s, 3H; 3'-OAc), 2.04 (s, 3H; 3-OAc), 2.05 (s, 3H; 4-OAc), 2.05 (s, 3H; 2'-OAc), 2.06 (s, 3H; 6'-OAc), 2.22 (s, 3H; 2-OAc), 3.49 (ddd, J = 10.0, 4.2, $2.5 \mathrm{~Hz}, 1 \mathrm{H}$; 5-H), 3.92 (dd, $J=12.3,2.5 \mathrm{~Hz}, 1 \mathrm{H} ; 6 \mathrm{a}-\mathrm{H}), 3.99$ (ddd, $\left.J=10.1,5.2,2.9 \mathrm{~Hz}, 1 \mathrm{H}, 5^{\prime}-\mathrm{H}\right), 4.08$ (dd, $J=12.3,4.2 \mathrm{~Hz}, 1 \mathrm{H} ; 6 \mathrm{~b}-\mathrm{H}), 4.21\left(\mathrm{dd}, J=12.4,2.9 \mathrm{~Hz}, 1 \mathrm{H} ; 6 \mathrm{a}^{\prime}-\mathrm{H}\right), 4.24(\mathrm{dd}, J=12.4,5.2 \mathrm{~Hz}$, $\left.1 \mathrm{H} ; 6 \mathrm{~b}^{\prime}-\mathrm{H}\right), 5.17(\mathrm{dd}, J=10.1,9.4 \mathrm{~Hz}, 1 \mathrm{H} ; 4-\mathrm{H}), 5.18\left(\mathrm{dd}, J=10.0,9.3 \mathrm{~Hz}, 1 \mathrm{H} ; 4^{\prime}-\mathrm{H}\right), 5.24(\mathrm{~d}$, $\left.J=7.8 \mathrm{~Hz}, 1 \mathrm{H} ; 1^{\prime}-\mathrm{H}\right), 5.25(\mathrm{~d}, J=8.1 \mathrm{~Hz}, 1 \mathrm{H} ; 1-\mathrm{H}), 5.30(\mathrm{dd}, J=9.8,9.4 \mathrm{~Hz}, 1 \mathrm{H} ; 3-\mathrm{H}), 5.37$ $\left(\mathrm{dd}, J=9.8,9.3 \mathrm{~Hz}, 1 \mathrm{H} ; 3^{\prime}-\mathrm{H}\right), 5.43\left(\mathrm{dd}, J=9.8,7.8 \mathrm{~Hz}, 1 \mathrm{H} ; 2^{\prime}-\mathrm{H}\right), 5.45(\mathrm{dd}, J=9.7,8.1 \mathrm{~Hz}, 1 \mathrm{H}$; 2-H), 7.19 (s, 1H; 3-ArH), 7.47 (ddd, $J=8.3,6.8,1.2 \mathrm{~Hz}, 1 \mathrm{H} ; 6-\mathrm{ArH}), 7.54$ (ddd, $J=8.5,6.8$, $1.3 \mathrm{~Hz}, 1 \mathrm{H} ; 7-\mathrm{ArH}), 7.99$ (ddd, $J=8.3,1.3,0.6 \mathrm{~Hz}, 1 \mathrm{H} ; 5-\mathrm{ArH}), 8.06 \mathrm{ppm}(\mathrm{ddd}, J=8.5,1.2$, $0.6 \mathrm{~Hz}, 1 \mathrm{H} ; 8-\mathrm{ArH}) .{ }^{13} \mathrm{C}-\mathrm{NMR}\left(150 \mathrm{MHz}, \mathrm{CD}_{2} \mathrm{Cl}_{2}\right.$ ): $\delta=20.7$ (q; OAc-6), 20.8 (q; OAc-4'), 20.8 (q; OAc-3), 20.8 (2q; OAc-2', OAc-3'), 20.9 (q; OAc-4), 21.0 (q; OAc-6'), 21.1 (q; OAc-2), 32.3 $(\mathrm{q} ; \mathrm{t}-\mathrm{Bu}), 36.8(\mathrm{~s} ; \mathrm{t}-\mathrm{Bu}), 61.7(\mathrm{t} ; \mathrm{C}-6), 62.8\left(\mathrm{t} ; \mathrm{C}-6^{\prime}\right), 68.6(\mathrm{~d} ; \mathrm{C}-4), 68.7\left(\mathrm{~d} ; \mathrm{C}-4^{\prime}\right), 71.3\left(\mathrm{~d} ; \mathrm{C}-2^{\prime}\right)$, 72.0 (C-5), 72.3 (d; C-2), $72.7\left(\mathrm{~d} ; \mathrm{C}-3^{\prime}\right), 72.8$ (d; C-5'), $73.1(\mathrm{~d} ; \mathrm{C}-3), 100.2\left(\mathrm{~d} ; \mathrm{C}-1^{\prime}\right), 100.8$ (d; C-1), 111.1 (d; ArC-3), 122.0 (d; ArC-5), 122.5 (d; ArC-8), 125.9 (d; ArC-6), 126.0 (s; ArC-4a), 126.9 (d; ArC-7), 128.4 (s; ArC-8a), 139.9 (s; ArC-2), 143.2 (s; ArC-1), 149.5 (s; ArC-4), 169.6 (s; OAc-2), 169.7 (s; OAc-2' $), 169.8$ (s; OAc-4), 169.9 (s; OAc-4'), 170.3 (s; OAc-3), 170.4 (s; OAc-3'), 170.6 (s; OAc-6), 170.8 ppm (s; OAc-6' $)$. IR (Film): v = 2965 (w), 1741 (s), 1434 (w), 1369 (m), 1220 (s), $1034 \mathrm{~cm}^{-1}$ (s). HRMS (ESI-Q-TOF): $m / z$ Calcd for $\mathrm{C}_{42} \mathrm{H}_{52} \mathrm{NaO}_{20}$ $\left[\mathrm{M}+\mathrm{Na}^{+}\right]$: 899.2944; Found: 899.2949. Anal. Calcd for (\%) $\mathrm{C}_{42} \mathrm{H}_{52} \mathrm{O}_{20}$ (876.86): C 57.53, $\mathrm{H}$ 5.98; Found: C 57.37, H 6.06.

\subsection{Photooxygenation of Carbohydrate-Substituted Naphthalenes $\mathbf{1}$ to Endoperoxides $\mathbf{2}$}

A solution of naphthalene $1(0.15 \mathrm{mmol})$ and sensitizer tetraphenylporphyrin (TPP, $1.0 \mathrm{mg})$ in $\mathrm{CD}_{2} \mathrm{Cl}_{2}(3.0 \mathrm{~mL})$ was cooled to $-70{ }^{\circ} \mathrm{C}$, oxygen was slowly bubbled through the solution, and the mixture was irradiated externally with a sodium lamp (400 W) for $2 \mathrm{~h}$. NMR spectra (500 MHz), which were directly measured from the crude products at $-70{ }^{\circ} \mathrm{C}$, showed complete conversion and the sole formation of endoperoxides 2 . The diastereomeric ratios $(d r)$ could be easily determined by integration of the distinctive signals of $\mathrm{H}^{3}$, which are separated by $0.2 \mathrm{ppm}$ (for NMR spectra, see Supplementary Materials; the characteristic chemical shifts of the minor isomers are marked bold). The reaction of $t \mathrm{Bu}$ derivative $\mathbf{1 f}$ with ${ }^{1} \mathrm{O}_{2}$ was conducted on a preparative scale as well. Thus, naphthalene 1f $(877 \mathrm{mg}, 1.0 \mathrm{mmol})$ and TPP $(15 \mathrm{mg})$ were dissolved in dichloromethane $(100 \mathrm{~mL})$ and the solution was photooxygenated at $-70{ }^{\circ} \mathrm{C}$ for $2 \mathrm{~h}$, as described above. The solvent was removed in vacuo and direct column chromatography (hexanes/EtOAc 1:1) at room temperature afforded endoperoxide $1 S, 4 R-2 \mathrm{f}(417 \mathrm{mg}, 46 \%)$ and $1 R, 4 S-2 \mathrm{f}$ (373 $\mathrm{mg}$, $41 \%$ ) in pure form.

Endoperoxide 2b. $R_{f}=0.56$ (PE/EtOAc 3:1). ${ }^{1} \mathrm{H}-\mathrm{NMR}\left(500 \mathrm{MHz}, \mathrm{CD}_{2} \mathrm{Cl}_{2},-78{ }^{\circ} \mathrm{C}\right): \delta=1.93$ (s, 3H; OAc), 1.99 (s, 3H; OAc), 1.99 (s, 3H; OAc), 2.00 (s, 6H; OAc), 2.00 (s, 3H; OAc), 2.05 (s, 3H; OAc), 2.06 (s, 3H; OAc), 3.84 (ddd, $J=9.9,4.7,2.1 \mathrm{~Hz}, 1 \mathrm{H} ; 5-\mathrm{H}), 3.88$ (ddd, J = 10.0, 3.8, $\left.2.4 \mathrm{~Hz}, 1 \mathrm{H} ; 5^{\prime}-\mathrm{H}\right), 4.01\left(\mathrm{dd}, J=12.5,2.4 \mathrm{~Hz}, 1 \mathrm{H} ; 6_{\mathrm{a}}{ }^{\prime}-\mathrm{H}\right), 4.06(\mathrm{dd}, J=12.5,2.1 \mathrm{~Hz}, 1 \mathrm{H}$; $\left.6_{\mathrm{a}}-\mathrm{H}\right), 4.28\left(\mathrm{~m}, 2 \mathrm{H} ; 6_{\mathrm{b}}-\mathrm{H}, 6_{\mathrm{b}}{ }^{\prime}-\mathrm{H}\right), 5.13\left(\mathrm{dd}, J=10.0,9.7 \mathrm{~Hz}, 1 \mathrm{H} ; 4^{\prime}-\mathrm{H}\right), 5.16(\mathrm{dd}, J=9.9,9.5 \mathrm{~Hz}$, $1 \mathrm{H} ; 4-\mathrm{H}), 5.19-5.31$ (m, 6H; 1-H, 1'-H, 2-H, 2'-H, 3-H, 3'-H); 6.94 (d, J = 9.3 Hz, 1H; 3-ArH), $7.16(\mathrm{~d}, J=9.3 \mathrm{~Hz}, 1 \mathrm{H} ; 2-\mathrm{ArH}), 7.28-7.33$ (m, 2H; 6-ArH, 7-ArH), 7.35-7.39 (m, 1H; 8-ArH), 7.43-7.46 ppm (m, 1H; 5-ArH). ${ }^{13} \mathrm{C}-\mathrm{NMR}\left(125 \mathrm{MHz}, \mathrm{CD}_{2} \mathrm{Cl}_{2},-78{ }^{\circ} \mathrm{C}\right): \delta=20.6$ (q; 2 x OAc), 20.6 (q; $3 \times$ OAc), 20.7 (q; 2 x OAc), 20.7 (q; OAc), $61.0(\mathrm{t} ; \mathrm{C}-6), 61.1\left(\mathrm{t} ; \mathrm{C}-6^{\prime}\right), 66.5(\mathrm{~d} ; \mathrm{C}-4)$, $66.8\left(\mathrm{~d} ; \mathrm{C}-4^{\prime}\right), 69.4\left(\mathrm{~d} ; \mathrm{C}-2^{\prime}\right), 70.1(\mathrm{~d} ; \mathrm{C}-2), 70.9\left(\mathrm{~d} ; \mathrm{C}-3^{\prime}\right), 71.2(\mathrm{~d} ; \mathrm{C}-3), 71.5\left(\mathrm{~d} ; \mathrm{C}^{\prime} 5^{\prime}\right), 71.6(\mathrm{~d}$; C-5), $96.5\left(\mathrm{~d} ; \mathrm{C}-1^{\prime}\right), 96.6$ (d; C-1), 101.7 (s; ArC-1), 102.8 (s; ArC-4), 119.2 (d; ArC-8), 119.4 (d; ArC-5), 127.5 (d; ArC-6), 127.6 (d; ArC-7), 133.3 (s; ArC-4a), 135.5 (d; ArC-2), 135.9 (s; ArC-8a), 136.0 (d; ArC-3), 169.1 (s; OAc), 169.4 (s; OAc), 169.4 (s; OAc), 169.5 (s; OAc), 170.0 (s; OAc), 170.2 (s; OAc), 170.6 (s; OAc-6), 170.6 ppm (s; OAc-6'). HRMS (ESI-Q-TOF): $m / z$ Calcd for $\mathrm{C}_{38} \mathrm{H}_{45} \mathrm{O}_{22}\left[\mathrm{M}+\mathrm{H}^{+}\right]$: 853.2402; Found: 853.2427.

Endoperoxide 2c. $R_{f}=0.51$ (PE/EtOAc 3:1). ${ }^{1} \mathrm{H}-\mathrm{NMR}\left(500 \mathrm{MHz}, \mathrm{CD}_{2} \mathrm{Cl}_{2},-78{ }^{\circ} \mathrm{C}\right): \delta=1.81$ (d, $J=1.4 \mathrm{~Hz}, 3 \mathrm{H} ; \mathrm{Me}$ ), 1.94 (s, 3H; OAc), 1.98 (s, 3H; OAc), 1.99 (s, 3H; OAc), 2.00 (s, 3H; 
OAc), 2.00 (s, 3H; OAc), 2.04 (s, 3H; 6'-OAc), 2.06 (s, 3H; OAc), 2.15 (s, 3H; 6-OAc), 3.82 (ddd, $J=9.7,5.4,2.1 \mathrm{~Hz}, 1 \mathrm{H} ; 5-\mathrm{H}), 3.89\left(\mathrm{ddd}, J=9.8,5.4,2.3 \mathrm{~Hz}, 1 \mathrm{H} ; 5^{\prime}-\mathrm{H}\right), 4.07$ (dd, $J=12.5$, $\left.2.3 \mathrm{~Hz}, 1 \mathrm{H} ; 6_{\mathrm{a}}{ }^{\prime}-\mathrm{H}\right), 4.13\left(\mathrm{dd}, J=12.3,2.1 \mathrm{~Hz}, 1 \mathrm{H} ; 6_{\mathrm{a}}-\mathrm{H}\right), 4.19-4.25\left(\mathrm{~m}, 2 \mathrm{H} ; 6_{\mathrm{b}}-\mathrm{H}, 6_{\mathrm{b}}{ }^{\prime}-\mathrm{H}\right), 5.05$ $\left(\mathrm{dd}, J=9.8,9.7 \mathrm{~Hz}, 1 \mathrm{H} ; 4^{\prime}-\mathrm{H}\right), 5.15(\mathrm{dd}, J=9.8,9.7 \mathrm{~Hz}, 1 \mathrm{H} ; 4-\mathrm{H}), 5.13-5.33(\mathrm{~m}, 6 \mathrm{H} ; 2-\mathrm{H}$, 1-H, 2'-H, 1'-H, 3-H, 3'-H), 6.43 (d, J = $1.5 \mathrm{~Hz}, 1 \mathrm{H} ; 3-\mathrm{ArH}), 6.61$ (d, J = $1.4 \mathrm{~Hz}, 1 \mathrm{H} ; 3-\mathrm{ArH})$, 7.26 (ddd, $J=7.4,7.3,1.2 \mathrm{~Hz}, 1 \mathrm{H} ; 7-\mathrm{ArH}), 7.31$ (ddd, $J=7.4,7.4,1.2 \mathrm{~Hz}, 1 \mathrm{H} ; 6-\mathrm{ArH}), 7.34$ (dd, $J=7.4,1.3 \mathrm{~Hz}, 1 \mathrm{H}$; 5-ArH, 6-ArH, 7-ArH), 7.39-7.40 (m, 1H; 5-ArH), 7.53-7.55 (m, 1H; 8-ArH), $7.85 \mathrm{ppm}(\mathrm{dd}, J=7.3,1.2 \mathrm{~Hz}, 1 \mathrm{H} ; 8-\mathrm{ArH}) .{ }^{13} \mathrm{C}-\mathrm{NMR}\left(125 \mathrm{MHz}, \mathrm{CD}_{2} \mathrm{Cl}_{2},-78{ }^{\circ} \mathrm{C}\right)^{\mathrm{a}}$ : $\delta=14.7$ (q; Me), 20.6 (q; $2 \times$ OAc), 20.6 (q; $3 \times$ OAc), 20.7 (q; OAc), 20.8 (q; OAc), 21.0 (q; OAc), $61.4\left(\mathrm{t} ; \mathrm{C}^{\prime} 6^{\prime}\right), 62.1(\mathrm{t} ; \mathrm{C}-6), 67.0(\mathrm{~d} ; \mathrm{C}-4), 67.4\left(\mathrm{~d} ; \mathrm{C}-4^{\prime}\right), 69.5\left(\mathrm{~d} ; \mathrm{C}-2^{\prime}\right), 69.8(\mathrm{~d} ; \mathrm{C}-2), 70.7$ (d; C-3), $70.9\left(\mathrm{~d} ; \mathrm{C}^{\prime} 3^{\prime}\right), 71.5\left(\mathrm{~d} ; \mathrm{C}-5^{\prime}\right), 71.7$ (d; C-5), 96.1 (2d; C-1, C-1'), 101.7 (s; ArC-4), 104.0 (s; ArC-1), 118.6 (d; ArC-5), 120.3 (d; ArC-8), 125.2 (d; ArC-3), 126.9 (d; ArC-7), 127.7 (d; ArC-6), 134.0 (s; ArC-8a), 138.8 (s; ArC-4a), 149.1 (d; ArC-2), 169.3 (s; OAc), 169.5 (s; OAc), 169.6 (s; OAc), 169.7 (s; OAc), 169.9 (s; OAc), 170.0 (s; OAc), 170.5 (s; OAc-6'), 170.6 ppm (s; OAc-6).

Endoperoxide 2d. $R_{f}=0.44$ (PE/EtOAc 2:1). ${ }^{1} \mathrm{H}-\mathrm{NMR}\left(500 \mathrm{MHz}, \mathrm{CD}_{2} \mathrm{Cl}_{2},-20{ }^{\circ} \mathrm{C}\right): \delta=0.97$ (d, J = 7.2 Hz, 3H; $\mathrm{CH}_{3}$ ), 1.97 (s, 3H; OAc), 1.99 (s, 3H; OAc), 2.00 (2s, 6H; 2 x OAc), 2.02 (s, 3H; OAc), 2.03 (s, 3H; OAc), 2.06 (s, 3H; 6'-OAc), 2.14 (s, 3H; 6-OAc), 2.73 (dq; J = 7.2, $1.7 \mathrm{~Hz}, 2 \mathrm{H} ; \mathrm{CH}_{2}$ ), 3.82 (ddd, $\left.J=10.0,6.0,2.2 \mathrm{~Hz}, 1 \mathrm{H} ; 5-\mathrm{H}\right), 3.91$ (ddd, $J=10.0,6.5,2.1 \mathrm{~Hz}$, $\left.1 \mathrm{H} ; 5^{\prime}-\mathrm{H}\right), 4.12\left(\mathrm{dd}, J=12.2,2.1 \mathrm{~Hz}, 1 \mathrm{H} ; 6_{\mathrm{a}}{ }^{\prime}-\mathrm{H}\right), 4.19\left(\mathrm{dd}, J=12.5,6.0 \mathrm{~Hz}, 1 \mathrm{H} ; 6_{\mathrm{b}}-\mathrm{H}\right), 4.20$ $\left(\mathrm{dd}, J=12.5,2.2 \mathrm{~Hz}, 1 \mathrm{H} ; 6_{\mathrm{a}}-\mathrm{H}\right), 4.27\left(\mathrm{dd}, J=12.2,6.5 \mathrm{~Hz}, 1 \mathrm{H} ; 6^{\prime}{ }_{\mathrm{b}}-\mathrm{H}\right), 5.06-5.11(\mathrm{~m}, 2 \mathrm{H}$; 4-H, $\left.4^{\prime}-\mathrm{H}\right), 5.16(\mathrm{dd}, \mathrm{J}=9.8,7.8 \mathrm{~Hz}, 1 \mathrm{H} ; 2-\mathrm{H}), 5.17-5.26\left(\mathrm{~m}, 4 \mathrm{H} ; 2^{\prime}-\mathrm{H}, 3^{\prime}-\mathrm{H}, 1-\mathrm{H}, 1^{\prime}-\mathrm{H}\right) ; 5.31$ $\left(\mathrm{dd}, J=9.6,9.0 \mathrm{~Hz}, 1 \mathrm{H} ; 3^{\prime}-\mathrm{H}\right), 6.31$ (s, 1H; 3-ArH), 6.54 (t, $\left.J=1.7 \mathrm{~Hz}, 1 \mathrm{H} ; 3-\mathrm{ArH}\right), 7.26$ (ddd, $J=7.6,7.5,1.0 \mathrm{~Hz}, 1 \mathrm{H} ; 7-\mathrm{ArH}), 7.32$ (ddd, $J=7.5,7.4,0.9 \mathrm{~Hz}, 1 \mathrm{H} ;$ 6-ArH, 6-ArH, 7-ArH), 7.38 (dd, $J=7.4,1.0 \mathrm{~Hz}, 1 \mathrm{H}$; 5-ArH), 7.45-7.47 (m, 1H; 5-ArH), 7.55-7.57 (m, 1H; 8-ArH), $7.88 \mathrm{ppm}(\mathrm{dd}, J=7.6,0.9 \mathrm{~Hz}, 1 \mathrm{H} ; 8-\mathrm{ArH}) .{ }^{13} \mathrm{C}-\mathrm{NMR}\left(125 \mathrm{MHz}, \mathrm{CD}_{2} \mathrm{Cl}_{2},-20{ }^{\circ} \mathrm{C}\right): \delta=10.2$ (q; $\mathrm{CH}_{3}$ ), 20.7 (q; OAc), 20.7 (q; OAc), 20.7 (q; 2 x OAc), 20.7 (q; OAc), 20.8 (q; OAc), 20.9 (q; OAc), $21.0\left(\mathrm{q} ; \mathrm{CH}_{3}\right), 21.1(\mathrm{q} ; \mathrm{OAc}), 62.3\left(\mathrm{t} ; \mathrm{C}-6^{\prime}\right), 62.5(\mathrm{t} ; \mathrm{C}-6), 67.9\left(\mathrm{~d} ; \mathrm{C}-4^{\prime}\right), 68.1(\mathrm{~d} ; \mathrm{C}-4), 70.2$ $\left(\mathrm{d} ; \mathrm{C}-2^{\prime}\right), 70.6(\mathrm{~d} ; \mathrm{C}-2), 71.7\left(\mathrm{~d} ; \mathrm{C}-3^{\prime}\right), 71.8(\mathrm{~d} ; \mathrm{C}-3), 72.3\left(\mathrm{~d} ; \mathrm{C}-5^{\prime}\right), 72.4(\mathrm{~d} ; \mathrm{C}-5), 96.5(\mathrm{~d} ; \mathrm{C}-1)$, 96.7 (d; C-1'), 102.5 (s; ArC-4), 104.6 (s; ArC-1), 119.0 (d; ArC-5), 120.7 (d; ArC-8), 123.5 (d; ArC-3), 127.2 (d; ArC-7), 127.9 (d; ArC-6), 135.2 (s; ArC-8a), 139.6 (s; ArC-4a), 155.1 (d; ArC-2), 169.5 (s; OAc), 169.7 (s; OAc), 169.7 (s; OAc), 169.8 (s; OAc), 170.0 (s; OAc), 170.1 (s; OAc), 170.6 (s; OAc-6), 170.7 ppm (s; OAc-6').

Endoperoxide 2e. $R_{f}=0.47$ (PE/EtOAc 2:1). ${ }^{1} \mathrm{H}-\mathrm{NMR}\left(500 \mathrm{MHz}, \mathrm{CD}_{2} \mathrm{Cl}_{2},-20{ }^{\circ} \mathrm{C}\right): \delta=0.90$ $\left(\mathrm{d}, \mathrm{J}=6.7 \mathrm{~Hz}, 3 \mathrm{H} ; \mathrm{CH}_{3}\right), 1.04\left(\mathrm{~d}, \mathrm{~J}=6.8 \mathrm{~Hz}, 3 \mathrm{H} ; \mathrm{CH}_{3}\right), 1.97$ (s, 3H; OAc), 1.99 (s, 3H; OAc), 2.00 (s, 3H; OAc), 2.00 (s, 3H; OAc), 2.02 (s, 3H; OAc), 2.03 (s, 3H; 6'-OAc), 2.05 (s, 3H; 2-OAc), 2.14 (s, 3H; 6-OAc), 2.73 (dsept; $J=6.8,1.4 \mathrm{~Hz}, 1 \mathrm{H} ; \mathrm{CH}$ ), 3.83 (ddd, $J=10.1,6.5$, $2.4 \mathrm{~Hz}, 1 \mathrm{H} ; 5-\mathrm{H}), 3.91\left(\mathrm{ddd}, J=10.0,6.0,2.1 \mathrm{~Hz}, 1 \mathrm{H} ; 5^{\prime}-\mathrm{H}\right), 4.12(\mathrm{dd}, J=12.3,2.1 \mathrm{~Hz}, 1 \mathrm{H}$; $\left.6_{\mathrm{a}}{ }^{\prime}-\mathrm{H}\right), 4.17\left(\mathrm{dd}, J=12.5,6.0 \mathrm{~Hz}, 1 \mathrm{H} ; 6_{\mathrm{b}}-\mathrm{H}\right), 4.19\left(\mathrm{dd}, J=12.5,2.4 \mathrm{~Hz}, 1 \mathrm{H} ; 6_{\mathrm{a}}-\mathrm{H}\right), 4.24(\mathrm{dd}$, $\left.J=12.3,6.5 \mathrm{~Hz}, 1 \mathrm{H} ; 6^{\prime}{ }_{\mathrm{b}}-\mathrm{H}\right), 5.08\left(\mathrm{dd}, J=10.0,9.6 \mathrm{~Hz}, 1 \mathrm{H} ; 4^{\prime}-\mathrm{H}\right), 5.10(\mathrm{dd}, J=10.1,9.2 \mathrm{~Hz}$, $1 \mathrm{H} ; 4-\mathrm{H}), 5.17(\mathrm{dd}, J=9.5,7.6 \mathrm{~Hz}, 1 \mathrm{H} ; 2-\mathrm{H}), 5.21$ (dd, $\left.J=9.7,7.8 \mathrm{~Hz}, 1 \mathrm{H} ; 2^{\prime}-\mathrm{H}\right), 5.22-5.27$ (m, 3H; 3-H, 1-H, 1'-H); $5.31\left(\mathrm{dd}, J=9.7,9.6 \mathrm{~Hz}, 1 \mathrm{H} ; 3^{\prime}-\mathrm{H}\right), 6.25$ (d, J = 1.6 Hz, 1H; 3-ArH), $6.50(\mathrm{~d}, J=1.4 \mathrm{~Hz}, 1 \mathrm{H} ; 3-\mathrm{ArH}), 7.25$ (ddd, $J=7.6,7.5,1.2 \mathrm{~Hz}, 1 \mathrm{H} ; 7-\mathrm{ArH}), 7.31$ (ddd, $J=7.5$, 7.5, 0.9 Hz, 1H; 6-ArH, 6-ArH, 7-ArH), 7.38 (dd, J = 7.4, 1.2 Hz, 1H; 5-ArH), 7.45-7.47 (m, 1H; 5-ArH), 7.59-7.61 (m, 1H; 8-ArH), 7.89 ppm (dd, $J=7.4,1.0$ Hz, 1H; 8-ArH). ${ }^{13} \mathrm{C}-\mathrm{NMR}$ $\left(125 \mathrm{MHz}, \mathrm{CD}_{2} \mathrm{Cl}_{2},-20{ }^{\circ} \mathrm{C}\right): \delta=20.1\left(\mathrm{q} ; \mathrm{CH}_{3}\right), 20.7$ (q; 2 x OAc), 20.7 ( $; ; 2$ x OAc), 20.7 (q; OAc), 20.8 (q; OAc), 20.8 (q; $\mathrm{CH}_{3}$ ), 20.9 (q;OAc), 21.0 (q;OAc), $27.0(\mathrm{~d} ; \mathrm{CH}), 62.4\left(\mathrm{t} ; \mathrm{C}^{\prime} 6^{\prime}\right)$, $62.6(\mathrm{t} ; \mathrm{C}-6), 67.9\left(\mathrm{~d} ; \mathrm{C}-4^{\prime}\right), 68.1(\mathrm{~d} ; \mathrm{C}-4), 70.2\left(\mathrm{~d} ; \mathrm{C}-2^{\prime}\right), 70.7$ (d; C-2), 71.8 (d; C-3'), 71.9 (d; C-3), 72.3 (d; C-5'), 72.4 (d; C-5), 96.5 (d; C-1), 96.7 (d; C-1'), 102.4 (s; ArC-4), 104.9 (s; ArC-1), 119.1 (d; ArC-5), 120.5 (d; ArC-8), 122.4 (d; ArC-3), 127.2 (d; ArC-7), 127.9 (d; ArC-6), 135.7 (s; ArC-8a), 139.6 (s; ArC-4a), 159.3 (d; ArC-2), 169.5 (s; OAc), 169.7 (s; OAc), 169.7 (s; OAc), 169.7 (s; OAc), 170.0 (s; OAc), 170.1 (s; OAc), 170.7 (s; OAc-6), 170.7 ppm (s; OAc-6'). 
Endoperoxide 1S,4R-2f. $R_{f}=0.19$ (PE/EtOAc 1:1). $[\alpha]_{D}^{24}=+13\left(c 1.04, \mathrm{CHCl}_{3}\right) .{ }^{1} \mathrm{H}-\mathrm{NMR}$ $\left(600 \mathrm{MHz}, \mathrm{CD}_{2} \mathrm{Cl}_{2}\right): \delta=1.15\left(\mathrm{~s}, 9 \mathrm{H} ;{ }^{\mathrm{t}} \mathrm{Bu}\right), 1.98$ (s, 3H; $\left.3^{\prime}-\mathrm{OAc}\right), 1.99$ (s, 3H; 2-OAc), 2.01 (s, $3 \mathrm{H} ; 3-\mathrm{OAc}), 2.02$ (2s, 6H; 6'-OAc, $\left.4^{\prime}-\mathrm{OAc}\right), 2.03$ (s, 3H; 4-OAc), 2.06 (s, 3H; $\left.2^{\prime}-\mathrm{OAc}\right), 2.14$ (s, 3H; 6-OAc), 3.81 (ddd, $J=10.0,5.1,3.8 \mathrm{~Hz}, 1 \mathrm{H} ; 5-\mathrm{H}), 3.93(\mathrm{ddd}, J=10.1,4.5,3.9 \mathrm{~Hz}$, $\left.1 \mathrm{H} ; 5^{\prime}-\mathrm{H}\right), 4.15-4.18\left(\mathrm{~m}, 2 \mathrm{H} ; 6 \mathrm{a}^{\prime}-\mathrm{H}, 6 \mathrm{~b}^{\prime}-\mathrm{H}\right), 4.21-4.28(\mathrm{~m}, 2 \mathrm{H} ; 6 \mathrm{a}-\mathrm{H}, 6 \mathrm{~b}-\mathrm{H}), 5.09$ (dd, J = 10.1, $\left.9.4 \mathrm{~Hz}, 1 \mathrm{H} ; 4^{\prime}-\mathrm{H}\right), 5.13(\mathrm{dd}, J=10.0,9.3 \mathrm{~Hz}, 4-\mathrm{H}), 5.19-5.22\left(\mathrm{~m}, 3 \mathrm{H} ; 2^{\prime}-\mathrm{H}, 2-\mathrm{H}, 3^{\prime}-\mathrm{H}\right), 5.28(\mathrm{~d}$, $J=7.6 \mathrm{~Hz}, 1 \mathrm{H} ; 1-\mathrm{H}), 5.30\left(\mathrm{~d}, J=7.8 \mathrm{~Hz}, 1 \mathrm{H} ; 1^{\prime}-\mathrm{H}\right), 5.32(\mathrm{dd}, J=9.7,9.3 \mathrm{~Hz}, 1 \mathrm{H} ; 3-\mathrm{H}), 6.57(\mathrm{~s}$, $1 \mathrm{H} ; 3-\mathrm{ArH}), 7.25$ (ddd, $J=7.6,7.5,1.3 \mathrm{~Hz}, 1 \mathrm{H} ; 7-\mathrm{ArH}), 7.31$ (ddd, $J=7.6,7.5,1.1 \mathrm{~Hz}, 1 \mathrm{H}$; 6-ArH), 7.41 (ddd, $J=7.5,1.3,0.5 \mathrm{~Hz}, 1 \mathrm{H} ; 5-\mathrm{ArH}) .7 .94 \mathrm{ppm}(\mathrm{ddd}, J=7.5,1.1,0.5 \mathrm{~Hz}, 1 \mathrm{H}$; 8-ArH). ${ }^{13} \mathrm{C}-\mathrm{NMR}\left(150 \mathrm{MHz}, \mathrm{CD}_{2} \mathrm{Cl}_{2}\right.$ ): $\delta=20.8$ (q; OAc-3'), 20.8 (q; OAc-2), 20.8 (q; OAc-3), 20.8 (q; OAc-6' $\left.{ }^{\prime}\right), 20.9$ (q; OAc-4'), 20.9 (q; OAc-4), 21.0 (q; OAc-2'), 21.0 (q; OAc-6), 28.4 (q; $\left.{ }^{\mathrm{t}} \mathrm{Bu}\right), 35.4\left(\mathrm{~s} ;{ }^{\mathrm{t}} \mathrm{Bu}\right), 62.8\left(\mathrm{t} ; \mathrm{C}-6^{\prime}\right), 63.0(\mathrm{t} ; \mathrm{C}-6), 68.7\left(\mathrm{~d} ; \mathrm{C}-4^{\prime}\right), 68.7(\mathrm{~d} ; \mathrm{C}-4), 70.9(\mathrm{~d} ; \mathrm{C}-2), 71.4$ $\left(\mathrm{d} ; \mathrm{C}-2^{\prime}\right), 72.4(\mathrm{~d} ; \mathrm{C}-3), 72.9\left(\mathrm{~d} ; \mathrm{C}-5^{\prime}\right), 72.9\left(\mathrm{~d} ; \mathrm{C}-3^{\prime}\right), 72.9(\mathrm{~d} ; \mathrm{C}-5), 97.1\left(\mathrm{~d} ; \mathrm{C}-1^{\prime}\right), 97.3(\mathrm{~d} ; \mathrm{C}-1)$, 102.4 (s; ArC-4), 107.3 (s; ArC-1), 119.6 (d; ArC-5), 120.8 (d; ArC-8), 124.5 (d; ArC-3), 127.3 (d; ArC-7), 128.0 (d; ArC-6), 137.0 (s; ArC-8a), 140.2 (s; ArC-4a), 161.3 (s; ArC-2), 169.7 (2s; OAc-2, OAc-2'), 169.8 (s; OAc-4), 169.9 (s; OAc-4' $\left.{ }^{\prime}\right), 170.1$ (s; OAc-3'), 170.2 (s; OAc-3), 170.7 (s; OAc-6), 170.8 ppm (s; OAc-6'). IR (Film): v = 3387 (br), 2927 (w), 1579 (w), 1395 (m), $1266(\mathrm{w}), 1239$ (w), 1147 (m), 1072 (s), $1036 \mathrm{~cm}^{-1}$ (s). HRMS (ESI-Q-TOF): $m / z$ Calcd for $\mathrm{C}_{42} \mathrm{H}_{52} \mathrm{NaO}_{22}\left(\mathrm{M}+\mathrm{Na}^{+}\right)$: 931.2842; Found: 931.2842.

Endoperoxide $1 R, 4 S-2$ f. $R_{f}=0.12$ (PE/EtOAc 1:1). $[\alpha]_{D}^{24}=-2.5$ (c 1.01, $\left.\mathrm{CHCl}_{3}\right) .{ }^{1} \mathrm{H}-\mathrm{NMR}$ $\left(600 \mathrm{MHz}, \mathrm{CD}_{2} \mathrm{Cl}_{2}\right): \delta=1.15$ (s, 9H; $\left.{ }^{\mathrm{t}} \mathrm{Bu}\right), 1.98$ (s, 3H; 4-OAc), 1.99 (s, 3H; $\left.2^{\prime}-\mathrm{OAc}\right), 2.00$ (s, 3H; 3-OAc), 2.00 (s, 3H; 3'-OAc), 2.01 (s, 3H; 4'-OAc), 2.03 (s, 3H; 6-OAc), 2.05 (s, 3H; 6'-OAc), 2.14 (s, 3H; 2-OAc), 3.70 (ddd, $J=10.1,4.8,2.5 \mathrm{~Hz}, 1 \mathrm{H} ; 5-\mathrm{H}), 3.86$ (ddd, $J=10.0,4.5$, $\left.2.4 \mathrm{~Hz}, 1 \mathrm{H} ; 5^{\prime}-\mathrm{H}\right), 4.06(\mathrm{dd}, J=12.3,2.5 \mathrm{~Hz}, 1 \mathrm{H} ; 6 \mathrm{a}-\mathrm{H}), 4.08\left(\mathrm{dd}, J=12.4,2.4 \mathrm{~Hz}, 1 \mathrm{H} ; 6 \mathrm{a}^{\prime}-\mathrm{H}\right)$, $4.23(\mathrm{dd}, J=12.3,4.8 \mathrm{~Hz}, 1 \mathrm{H} ; 6 \mathrm{~b}-\mathrm{H}), 4.30\left(\mathrm{dd}, J=12.4,2.4 \mathrm{~Hz}, 6 \mathrm{~b}^{\prime}-\mathrm{H}\right), 5.13(\mathrm{dd}, J=10.1$, $9.2 \mathrm{~Hz}, 1 \mathrm{H} ; 4-\mathrm{H}), 5.15\left(\mathrm{dd}, J=10.0,9.5 \mathrm{~Hz}, 1 \mathrm{H} ; 4^{\prime}-\mathrm{H}\right), 5.23\left(\mathrm{dd}, J=9.7,8.0 \mathrm{~Hz}, 1 \mathrm{H} ; 2^{\prime}-\mathrm{H}\right)$, $5.28(\mathrm{dd}, J=9.6,9.2 \mathrm{~Hz}, 1 \mathrm{H} ; 3-\mathrm{H}), 5.29\left(\mathrm{~d}, J=8.0 \mathrm{~Hz}, 1 \mathrm{H}: 1^{\prime}-\mathrm{H}\right), 5.31(\mathrm{dd}, J=9.7,9.5 \mathrm{~Hz}, 1 \mathrm{H}$; $\left.3^{\prime}-\mathrm{H}\right), 5.37(\mathrm{~d}, J=8.1 \mathrm{~Hz}, 1 \mathrm{H} ; 1-\mathrm{H}), 5.37(\mathrm{dd}, J=9.6,8.1 \mathrm{~Hz}, 1 \mathrm{H} ; 2-\mathrm{H}), 6.28(\mathrm{~s}, 1 \mathrm{H} ; 3-\mathrm{ArH})$, 7.30-7.33 (m, 2H; 6-ArH, 7-ArH), 7.47-7.49 (m, 1H; 5-ArH), 7.64-7.67 ppm (m, 1H; 8-ArH). ${ }^{13} \mathrm{C}-\mathrm{NMR}\left(150 \mathrm{MHz}, \mathrm{CD}_{2} \mathrm{Cl}_{2}\right.$ ): $\delta=20.7$ (q; OAc-4), 20.7 (3q; OAc-2', OAc-3, OAc-4'), 20.8 (q; OAc-3'), $20.8(\mathrm{q} ; \mathrm{OAc}-6), 20.8\left(\mathrm{q} ; \mathrm{OAc}^{\prime}{ }^{\prime}\right), 20.9(\mathrm{q} ; \mathrm{OAc}-2), 28.2\left(\mathrm{q} ;{ }^{\mathrm{t}} \mathrm{Bu}\right), 35.5\left(\mathrm{~s} ;{ }^{\mathrm{t}} \mathrm{Bu}\right), 61.9(\mathrm{t}$; C-6), $62.0\left(\mathrm{t} ; \mathrm{C}-6^{\prime}\right), 68.2\left(\mathrm{~d} ; \mathrm{C}-4^{\prime}\right), 68.6(\mathrm{~d} ; \mathrm{C}-4), 71.6\left(\mathrm{~d} ; \mathrm{C}-2^{\prime}\right), 71.8(\mathrm{~d} ; \mathrm{C}-2), 72.5(\mathrm{~d} ; \mathrm{C}-5), 72.8$ (2d; C-5', C-3'), $73.2(\mathrm{~d} ; \mathrm{C}-3), 97.1(\mathrm{~d} ; \mathrm{C}-1), 97.2\left(\mathrm{~d} ; \mathrm{C}-1^{\prime}\right), 103.2(\mathrm{~s} ; \mathrm{ArC}-4), 107.3(\mathrm{~s} ; \mathrm{ArC}-1)$, 119.9 (d; ArC-5), 120.1 (d; ArC-8), 124.6 (d; ArC-3), 127.3 (d; ArC-7), 128.0 (d; ArC-6), 136.7 (s; ArC-8a), 140.2 (s; ArC-4a), 161.5 (s; ArC-2), 169.1 (s; OAc-2), $169.2(\mathrm{~s} ;$ OAc-2'), 169.7 (s; OAc-4'), 169.7 (s; OAc-4), 170.3 (s; OAc-3), 170.3 (s; OAc-3'), 170.7 (s; OAc-6), 170.7 ppm (s; OAc-6'). IR (Film): v = 3387 (br), $2927(\mathrm{w}), 1579(\mathrm{w}), 1395(\mathrm{~m}), 1266(\mathrm{w}), 1239(\mathrm{w}), 1147$ (m), 1072 (s), $1036 \mathrm{~cm}^{-1}$ (s). HRMS (ESI-Q-TOF): $m / z$ Calcd for $\mathrm{C}_{42} \mathrm{H}_{52} \mathrm{NaO}_{22}\left(\mathrm{M}+\mathrm{Na}^{+}\right)$: 931.2842; Found: 931.2852.

\subsection{Thermolysis of Endoperoxides 2 and Determination of Singlet Oxygen Yield}

The half lifetimes of endoperoxides 2 were determined by measuring UV/VIS spectra at different temperatures and observing the increasing absorption band of the corresponding naphthalene 1 at $290-320 \mathrm{~nm}$. The singlet oxygen yield was determined by trapping with tetramethylethylene. Thus, the cold solution of crude endoperoxide $\mathbf{2 b}$ was purged with argon for $2 \mathrm{~min}, 20$ equiv. of tetramethylethylene were added, and the solution was warmed to room temperature within $8 \mathrm{~h}$. The ${ }^{1} \mathrm{O}_{2}$ yield was then determined by ${ }^{1} \mathrm{H}-\mathrm{NMR}$ integrals of the formed hydroperoxide.

\subsection{Transformation of Endoperoxide $1 S, 4 R-\mathbf{2 f}$ into Enantiomerically Pure Epoxide $2 S, 3 R-8$}

Endoperoxide $1 S, 4 R-2 \mathrm{f}(360 \mathrm{mg}, 0.4 \mathrm{mmol})$ was dissolved in dichloromethane $(10 \mathrm{~mL})$, cooled to $0{ }^{\circ} \mathrm{C}$, and a cold solution of $m$-chloroperoxybenzoic acid $(70 \mathrm{mg}, 0.4 \mathrm{mmol})$ in dichloromethane $(10 \mathrm{~mL})$ was added at $0{ }^{\circ} \mathrm{C}$. After $16 \mathrm{~h}$ at $0{ }^{\circ} \mathrm{C}$, TLC showed incomplete conversion and another amount of $m$-chloroperoxybenzoic acid $(35 \mathrm{mg}, 0.2 \mathrm{mmol})$ in 
dichloromethane $(5 \mathrm{~mL})$ was added. After 2 days at room temperature, the reaction mixture was extracted with a saturated solution of potassium carbonate $(2 \times 20 \mathrm{~mL})$, washed with water $(20 \mathrm{~mL})$, and dried over sodium sulfate. After removal of solvent in vacuo, the crude product was purified by column chromatography (hexanes/EtOAc 1:1) to afford epoxide syn-7 (280 mg, 76\%) as sole diastereomer.

$R_{f}=0.16\left(\mathrm{PE} /\right.$ EtOAc 1:1). $[\alpha]_{D}^{24}=+66\left(c\right.$ 0.99, $\left.\mathrm{CHCl}_{3}\right) .{ }^{1} \mathrm{H}-\mathrm{NMR}\left(400 \mathrm{MHz}, \mathrm{CDCl}_{3}\right)$ : $\delta=0.92\left(\mathrm{~s}, 9 \mathrm{H} ;{ }^{\mathrm{t}} \mathrm{Bu}\right), 1.99$ (s, 3H; OAc), 2.02 (s, 3H; OAc), 2.03 (s, 3H; OAc), 2.03 (s, 3H; OAc), 2.04 (s, 3H; OAc), 2.06 (s, 3H; OAc), 2.15 (s, 3H; OAc), 2.18 (s, 3H; OAc), 3.77-3.82 (m, 1H; 5-H), 3.92 (s, 1H; 3-H), 3.93-4.00 (m, 1H; 5'-H), 4.19-4.28 (m, 4H; 6a'-H, 6b'-H, 6a-H, 6b-H), 5.09-5.26 (m, 6H; 1'-H, 2-H, 2'-H, 3'-H, 4-H, 4'-H), 5.32-5.38 (m, 2H; 3-H, 1-H), 7.42-7.57 (m, 3-ArH; 5-ArH, 6-H, 7-ArH), 8.03-8.06 ppm (m, 8-ArH). ${ }^{13} \mathrm{C}-\mathrm{NMR}\left(100 \mathrm{MHz}, \mathrm{CDCl}_{3}\right)$ : $\delta=20.8$ (2q; OAc), 20.8 (3q; OAc), 20.8 (q; OAc), 21.0 (q;OAc), 21.0 (q;OAc), $26.5\left(\mathrm{q} ;{ }^{\mathrm{t}} \mathrm{Bu}\right.$ ), $32.9\left(\mathrm{~s} ;{ }^{\mathrm{t}} \mathrm{Bu}\right), 61.0(\mathrm{~d} ; \mathrm{C}-3), 62.5\left(\mathrm{t} ; \mathrm{C}-6^{\prime}\right), 62.8(\mathrm{t} ; \mathrm{C}-6), 68.6\left(\mathrm{~d} ; \mathrm{C}-4^{\prime}\right), 68.9(\mathrm{~d} ; \mathrm{C}-4), 70.9(\mathrm{~d} ; \mathrm{C}-2)$, $71.3\left(\mathrm{~d} ; \mathrm{C}-2^{\prime}\right), 71.4(\mathrm{~s} ; \operatorname{ArC}-2), 72.4(\mathrm{~d} ; \mathrm{C}-3), 72.6\left(\mathrm{~d} ; \mathrm{C}-5^{\prime}\right), 73.0\left(\mathrm{~d} ; \mathrm{C}-3^{\prime}\right), 73.1$ (d; C-5), 97.1 $\left(\mathrm{d} ; \mathrm{C}-1^{\prime}\right), 97.1$ (d; C-1), 100.1 (s; ArC-4), 106.4 (s; ArC-1), 121.3 (d; ArC-5), 123.5 (d; ArC-8), 129.1 (d; ArC-7), 129.3 (d; ArC-6), 137.0 (d; ArC-8a), 138.4 (s; ArC-4a),169.6 (s; OAc), 169.9 (3s; OAc), 170.1 (s; OAc), 170.2 (s; OAc), 170.7 (s; OAc), 170.7 ppm (s; OAc-6'). IR (Film): $v=3660(\mathrm{w}), 3501(\mathrm{w}), 1743$ (s), 1434 (m), 1364 (s), 1214 (s), 1037 (s). HRMS (ESI-Q-TOF): $m / z$ Calcd for $\mathrm{C}_{42} \mathrm{H}_{52} \mathrm{NaO}_{23}\left(\mathrm{M}+\mathrm{Na}^{+}\right)$: 947.2792, Found: 947.2790.

Epoxide syn-7 (190 mg, $0.2 \mathrm{mmol})$ was dissolved in dichloromethane $(10 \mathrm{~mL})$, and palladium on charcoal $(20 \mathrm{mg}, 5 \mathrm{~mol} \%$ ) was added. The solution was purged with hydrogen gas for 5 min, equipped with a balloon filled with hydrogen gas and hydrogenated under stirring at 1 bar for $16 \mathrm{~h}$. The solvent was removed in vacuo, and column chromatography (hexanes/EtOAc 1:1) afforded epoxide 8 (30 mg,63\%) as a white solid in enantiomerically pure form, which was confirmed by HPLC on a chiral phase (for chromatograms, see Figure 5).

M.p. $=61-63{ }^{\circ} \mathrm{C} . R_{f}=0.81(\mathrm{PE} / \mathrm{EtOAc} 1: 1) .[\alpha]_{D}^{25}=-22\left(c 1.00, \mathrm{CHCl}_{3}\right) .{ }^{1} \mathrm{H}-\mathrm{NMR}$ $\left(400 \mathrm{MHz} \mathrm{CDCl}_{3}\right): \delta=1.23\left(\mathrm{~s}, 9 \mathrm{H} ;{ }^{\mathrm{t}} \mathrm{Bu}\right), 3.99(\mathrm{~s}, 1 \mathrm{H} ; 3-\mathrm{H}), 7.67-7.76(\mathrm{~m}, 2 \mathrm{H} ; 5-\mathrm{H}, 6-\mathrm{H})$, 7.88-7.91 (m, 1H; 3-H, 5-H), 7.96-7.99 ppm (m, 1H; 8-H). ${ }^{13} \mathrm{C}-\mathrm{NMR}\left(100 \mathrm{MHz}, \mathrm{CDCl}_{3}\right)$ : $\delta=26.3(\mathrm{q} ; t-\mathrm{Bu}), 33.0(\mathrm{~s} ; \mathrm{t}-\mathrm{Bu}), 58.8(\mathrm{~d} ; \mathrm{C}-3), 68.4(\mathrm{~s} ; \mathrm{C}-2), 126.3(\mathrm{~d} ; \mathrm{C}-5), 127.8(\mathrm{~d} ; \mathrm{C}-8), 131.3$ (s; C-4a), 134.0 (d; C-6), 134.2 (s; C-8a), 134.7 (d; C-7), 191.5 (s; C-1), 192.7 ppm (s; C-4). IR (Film): v = 3484(w, br), 2962 (w), 2921 (w), 2877 (w), 1695 (s), 1596 (s), 1461 (m), 1341 (m), 1322 (m), 1299 (s), 1143 (s), 916 (s), 881 (s). LRMS (GC-TOF): $m / z$ Calcd for $\mathrm{C}_{14} \mathrm{H}_{14} \mathrm{O}_{3}\left(\mathrm{M}^{+}\right)$: 230.0943; Found: 230.0941.

\section{Conclusions}

In conclusion, we could control the [4+2] cycloaddition of singlet oxygen to naphthalenes by carbohydrates. Such a chiral auxiliary approach with sugars was hitherto unknown. The precursors are easily available from naphthoquinone and Schmidt's trichloroacetimidate in only 2-3 steps. The photooxygenations afforded endoperoxides (EPOs) as sole products, which were characterized by low-temperature NMR, but can reconvert to the parent naphthalenes under release of singlet oxygen. However, sterically demanding EPOs could be isolated by column chromatography in enatiomerically pure form at room temperature for the first time. Their absolute configurations were determined by a combination of NOESY measurements and theoretical calculations. We found a remarkable influence of substituents on the diastereoselectivities of the photooxygenations, ranging from no selectivity to up to $91: 9 d r$, which was rationalized by preferred rotamers of the carbohydrates. Thus, the sugars control the attack of singlet oxygen by steric and electrostatic repulsions. Finally, we could transfer the chiral information of the EPOs to an epoxide with high selectivity. After removal of the carbohydrates, we obtained a naphthoquinone epoxide in enantiomerically pure form, proven by HPLC on a chiral phase. This allowed the unequivocal determination of the absolute configurations of the EPOs. Furthermore, since the carbohydrates can be easily introduced, influence the selectivities of 
the photooxygenations, and are finally cleaved, the concept of a chiral auxiliary controlled $[4+2]$ cycloaddition of singlet oxygen to naphthalenes has been realized.

Supplementary Materials: The following are available online, S2-S5: ${ }^{1} \mathrm{H}$ - and ${ }^{13} \mathrm{C}-\mathrm{NMR}$ spectra of naphthoquinones 3, S6-S10: ${ }^{1} \mathrm{H}$ - and ${ }^{13} \mathrm{C}-\mathrm{NMR}$ spectra of carbohydrate-substituted naphthalenes $\mathbf{1}$, S11-S17: ${ }^{1} \mathrm{H}$ - and ${ }^{13} \mathrm{C}-\mathrm{NMR}$ spectra of endoperoxides 2, S18-S19: NOESY spectra of endoperoxides 2f, S20-S21: ${ }^{1} \mathrm{H}$ - and ${ }^{13} \mathrm{C}-\mathrm{NMR}$ spectra of epoxides 7 and 8, S22-S30: theoretical calculations.

Author Contributions: Conceptualization, T.L.; synthesis, M.B.; NMR evaluation, M.B.; calculations, M.B. and W.F.; HPLC analysis, W.F.; writing T.L.; funding acquisition, T.L. All authors have read and agreed to the published version of the manuscript.

Funding: This research was funded by the University of Potsdam, Germany.

Data Availability Statement: The data presented in this study are available in this article.

Acknowledgments: We thank Melanie Metz for the synthesis of some starting materials.

Conflicts of Interest: The authors declare no conflict of interest.

Sample Availability: Not available.

\section{References}

1. Nonell, S.; Flors, C. (Eds.) Singlet Oxygen; Comprehensive Series in Photochemical \& Photobiological Sciences; The Royal Society of Chemistry: London, UK, 2016; Volume 1, ISBN 978-1-78262-038-9.

2. Gorman, A.A.; Rodgers, M.A.J. Singlet molecular oxygen. Chem. Soc. Rev. 1981, 10, 205-231. [CrossRef]

3. Ogilby, P.R.; Foote, C.S. Chemistry of singlet oxygen. 42. Effect of solvent, solvent isotopic substitution, and temperature on the lifetime of singlet molecular oxygen. J. Am. Chem. Soc. 1983, 105, 3423-3430. [CrossRef]

4. Kuimova, M.K.; Yahioglu, G.; Ogilby, P.R. Singlet Oxygen in a Cell: Spatially Dependent Lifetimes and Quenching Rate Constants. J. Am. Chem. Soc. 2009, 131, 332-340. [CrossRef]

5. Stratakis, M.; Orfanopoulos, M. Regioselectivity in the Ene Reaction of Singlet Oxygen with Alkenes. Tetrahedron 2000, 56, 1595-1615. [CrossRef]

6. Prein, M.; Adam, W. The Schenck Ene Reaction: Diastereoselective Oxyfunctionalization with Singlet Oxygen in Synthetic Applications. Angew. Chem. (Int. Ed. Engl.) 1996, 35, 477-494. [CrossRef]

7. Adam, W.; Nestler, B. Photooxygenation of chiral allylic alcohols: Hydroxy-directed regio- and diastereoselective ene reaction of singlet oxygen. J. Am. Chem. Soc. 1992, 114, 6549-6550. [CrossRef]

8. Adam, W.; Nestler, B. Hydroxy-Directed Regio- and Diastereoselective Ene Reaction of Singlet Oxygen with Chiral Allylic Alcohols. J. Am. Chem. Soc. 1993, 115, 5041-5049. [CrossRef]

9. Adam, W.; Prein, M. Diastereoselective [4+2] cycloaddition of singlet oxygen in the photooxygenation of chiral naphthyl alcohols: Evidence for a hydroxy group-directing effect. J. Am. Chem. Soc. 1993, 115, 3766-3767. [CrossRef]

10. Adam, W.; Prein, M. $\pi$-Facial Diastereoselectivity in the [4+2] Cycloaddition of Singlet Oxygen as a Mechanistic Probe. Acc. Chem. Res. 1996, 29, 275-283. [CrossRef]

11. Adam, W.; Prein, M. Substituent effects in the diastereoselective [4+2] cycloaddition of chiral naphthalene derivatives with singlet oxygen. Tetrahedron Lett. 1994, 35, 4331-4334. [CrossRef]

12. Linker, T.; Fröhlich, L. Regio- and Diastereoselective Photooxygenation of Chiral 2,5-Cyclohexadiene-1-carboxylic Acids. Angew. Chem. Int. Ed. Engl. 1994, 33, 1971-1972. [CrossRef]

13. Linker, T.; Fröhlich, L. Substituent Effects in the Highly Regioselective and Diastereoselective Ene Reaction of Singlet Oxygen with Chiral Cyclohexadienes. J. Am. Chem. Soc. 1995, 117, 2694-2697. [CrossRef]

14. Sundén, H.; Engqvist, M.; Casas, J.; Ibrahem, I.; Córdova, A. Direct amino acid catalyzed asymmetric $\alpha$ oxidation of ketones with molecular oxygen. Angew. Chem. Int. Ed. 2004, 43, 6532-6535. [CrossRef]

15. Córdova, A.; Sundén, H.; Engqvist, M.; Ibrahem, I.; Casas, J. The Direct Amino Acid-Catalyzed Asymmetric Incorporation of Molecular Oxygen to Organic Compounds. J. Am. Chem. Soc. 2004, 126, 8914-8915. [CrossRef]

16. Walaszek, D.J.; Jawiczuk, M.; Durka, J.; Drapała, O.; Gryko, D. $\alpha$-Photooxygenation of chiral aldehydes with singlet oxygen. Beilstein J. Org. Chem. 2019, 15, 2076-2084. [CrossRef]

17. Joy, A.; Robbins, R.J.; Pitchumani, K.; Ramamurthy, V. Asymmetrically modified zeolite as a medium for enantioselective photoreactions: Reactions from spin forbidden excited states. Tetrahedron Lett. 1997, 38, 8825-8828. [CrossRef]

18. Griesbeck, A.G.; Bartoschek, A.; Neudörfl, J.; Miara, C. Stereoselectivity in Ene Reactions with Singlet Oxygen: Matrix Effects in Polymer Supports, Photooxygenation of Organic Salts and Asymmetric Synthesis. Photochem. Photobiol. 2006, 82, 1233-1240. [CrossRef]

19. Griesbeck, A.G.; Miranda, M.A.; Uhlig, J. Sweet chiral porphyrins as singlet oxygen sensitizers for asymmetric Type II photooxygenation. Photochem. Photobiol. Sci. 2011, 10, 1431-1435. [CrossRef] [PubMed] 
20. Wiegand, C.; Herdtweck, E.; Bach, T. Enantioselectivity in visible light-induced, singlet oxygen [2+4] cycloaddition reactions (type II photooxygenations) of 2-pyridones. Chem. Commun. 2012, 48, 10195-10197. [CrossRef] [PubMed]

21. Adam, W.; Brünker, H.-G.; Nestler, B. Photooxygenation of 2-alkenyl substituted chiral oxazolines: Stereochemical evidence favouring the cis effect in the regioselective ene reaction of singlet oxygen with $\alpha, \beta$-unsaturated carbonyl derivatives. Tetrahedron Lett. 1991, 32, 1957-1960. [CrossRef]

22. Dussault, P.H.; Woller, K.R.; Hillier, M.C. Stereoselective dioxygenation of enoates. Tetrahedron 1994, 50, 8929-8940. [CrossRef]

23. Fudickar, W.; Vorndran, K.; Linker, T. Auxiliary controlled singlet-oxygen ene reactions of cyclohexenes. Tetrahedron 2006, 62, 10639-10646. [CrossRef]

24. Adam, W.; Peters, K.; Peters, E.-M.; Schambony, S.B. Diastereoselective and Regioselective Singlet-Oxygen Ene Reaction of Oxazolidine-Substituted Alkenes: Control through Hydrogen Bonding Mediated by the Urea Functionality of Chiral Auxiliaries. J. Am. Chem. Soc. 2000, 122, 7610-7611. [CrossRef]

25. Adam, W.; Degen, H.G.; Krebs, O.; Saha-Möller, C.R. Efficient $\pi$-facial control in the ene reaction of nitrosoarene, triazolinedione, and singlet oxygen with tiglic amides of the bornane-derived sultam as chiral auxiliary. J. Am. Chem. Soc. 2002, 124, 12938-12939. [CrossRef] [PubMed]

26. Adam, W.; Bosio, S.G.; Turro, N.J. Highly diastereoselective dioxetane formation in the photooxygenation of enecarbamates with an oxazolidinone chiral auxiliary: Steric control in the [2+2] cycloaddition of singlet oxygen through conformational alignment. J. Am. Chem. Soc. 2002, 124, 8814-8815. [CrossRef] [PubMed]

27. Sivaguru, J.; Solomon, M.R.; Poon, T.; Jockusch, S.; Bosio, S.G.; Adam, W.; Turro, N.J. The reaction of singlet oxygen with enecarbamates: A mechanistic playground for investigating chemoselectivity, stereoselectivity, and vibratioselectivity of photooxidations. Acc. Chem. Res. 2008, 41, 387-400. [CrossRef] [PubMed]

28. Bánez Sanz, J.; Galisteo González, D.; López Sastre, J.A.; Rodríguez Amo, J.F.; Romero-Avila García, C.; Santos García, M.; Sanz Tejedor, M.A. Photooxygenation of alditol-1-C-yl derivatives of furan with singlet oxygen. Carbohydr. Res. 1996, 289, 179-188. [CrossRef]

29. Cermola, F.; Iesce, M.R.; Buonerba, G. Dye-Sensitized Photooxygenation of Furanosyl Furans: Synthesis of a New Pyridazine C-Nucleoside. J. Org. Chem. 2005, 70, 6503-6505. [CrossRef]

30. Cermola, F.; Iesce, M.R. Dye-sensitized photooxygenation of sugar-furans as synthetic strategy for novel C-nucleosides and functionalized exo-glycals. Tetrahedron 2006, 62, 10694-10699. [CrossRef]

31. Linker, T.; Sommermann, T.; Kahlenberg, F. The addition of malonates to glycals: A general and convenient method for the synthesis of 2-C-branched carbohydrates. J. Am. Chem. Soc. 1997, 119, 9377-9384. [CrossRef]

32. Elamparuthi, E.; Linker, T. Carbohydrate-2-deoxy-2-phosphonates: Simple synthesis and horner-emmons reaction. Angew. Chem. Int. Ed. 2009, 48, 1853-1855. [CrossRef]

33. Mai-Linde, Y.; Linker, T. Radical Clock Probes to Determine Carbohydrate Radical Stabilities. Org. Lett. 2020, $22,1525-1529$. [CrossRef] [PubMed]

34. Zehm, D.; Fudickar, W.; Linker, T. Molecular switches flipped by oxygen. Angew. Chem. Int. Ed. 2007, 46, 7689-7692. [CrossRef] [PubMed]

35. Klaper, M.; Fudickar, W.; Linker, T. Role of Distance in Singlet Oxygen Applications: A Model System. J. Am. Chem. Soc. 2016, 138, 7024-7029. [CrossRef] [PubMed]

36. Fudickar, W.; Linker, T. Singlet Oxygen Release of Singlet Oxygen from Aromatic Endoperoxides by Chemical Triggers Angewandte. Angew. Chem. Int. Ed. 2018, 57, 12971-12975. [CrossRef] [PubMed]

37. He, Y.-Q.; Fudickar, W.; Tang, J.-H.; Wang, H.; Li, X.; Han, J.; Wang, Z.; Liu, M.; Zhong, Y.-W.; Linker, T.; et al. Capture and Release of Singlet Oxygen in Coordination-Driven Self-Assembled Organoplatinum(II) Metallacycles. J. Am. Chem. Soc. 2020, 142, 2601-2608. [CrossRef] [PubMed]

38. Wasserman, H.H.; Larsen, D.L. Formation of 1-4-endoperoxides from the dye-sensitized photooxygenation of alkyl-naphthalenes. J. Chem. Soc. Chem. Commun. 1972, 253-254. [CrossRef]

39. Bauch, M.; Krtitschka, A.; Linker, T. Photooxygenation of oxygen-substituted naphthalenes. J. Phys. Org. Chem. 2017, 30, 1-11. [CrossRef]

40. Turro, N.J.; Chow, M.F. Mechanism of thermolysis of endoperoxides of aromatic compounds. Activation parameters, magnetic field, and magnetic isotope effects. J. Am. Chem. Soc. 1981, 103, 7218-7224. [CrossRef]

41. Aubry, J.-M.; Pierlot, C.; Rigaudy, J.; Schmidt, R. Reversible Binding of Oxygen to Aromatic Compounds. Acc. Chem. Res. 2003, 36, 668-675. [CrossRef]

42. Fudickar, W.; Linker, T. Release of Singlet Oxygen from Organic Peroxides under Mild Conditions. ChemPhotoChem 2018, 2, 548-558. [CrossRef]

43. Klaper, M.; Linker, T. New singlet oxygen donors based on naphthalenes: Synthesis, physical chemical data, and improved stability. Chem. Eur. J. 2015, 21, 8569-8577. [CrossRef]

44. Klaper, M.; Linker, T. Intramolecular Transfer of Singlet Oxygen. J. Am. Chem. Soc. 2015, 137, 13744-13747. [CrossRef] [PubMed]

45. Coppa, F.; Fontana, F.; Lazzarini, E.; Minisci, F. A New Selective Method for the Homolytic Alkylation and Carboxylation of Quinones by Monoesters of Oxalic Acid. Chem. Lett. 1992, 21, 1299-1302. [CrossRef] 
46. Fujii, S.; Shimizu, A.; Takeda, N.; Oguchi, K.; Katsurai, T.; Shirakawa, H.; Komai, M.; Kagechika, H. Systematic synthesis and anti-inflammatory activity of $\omega$-carboxylated menaquinone derivatives-Investigations on identified and putative vitamin $\mathrm{K} 2$ metabolites. Bioorg. Med. Chem. 2015, 23, 2344-2352. [CrossRef] [PubMed]

47. Schmidt, R.R. New Methods for the Synthesis of Glycosides and Oligosaccharides-Are There Alternatives to the Koenigs-Knorr Method? Angew. Chem. Int. Ed. Engl. 1986, 25, 212-235. [CrossRef]

48. Tietze, L.F.; Schmuck, K.; Schuster, H.J.; Müller, M.; Schuberth, I. Synthesis and biological evaluation of prodrugs based on the natural antibiotic duocarmycin for use in ADEPT and PMT. Chem. Eur. J. 2011, 17, 1922-1929. [CrossRef]

49. Beckhaus, H.-D. Sf Parameters-A Measure of the Front Strain of Alkyl Groups. Angew. Chem. Int. Ed. Engl. 1978, 17, 593-594. [CrossRef]

50. Engelhardt, U.; Sarkar, A.; Linker, T. Efficient enantioselective total synthesis of (-)-epipodophyllotoxin. Angew. Chem. Int. Ed. 2003, 42, 2487-2489. [CrossRef]

51. Sasaoka, M.; Hart, H. Endoperoxides of Naphthalenes. Synthesis and Reactions of Substituted 2,3-Epoxynaphthalene 1,4Endoperoxides. J. Org. Chem. 1979, 44, 368-374. [CrossRef]

52. Bunge, A.; Hamann, H.-J.; McCalmont, E.; Liebscher, J. Enantioselective epoxidation of 2-substituted 1,4-naphthoquinones using gem-dihydroperoxides. Tetrahedron Lett. 2009, 50, 4629-4632. [CrossRef]

53. Fioroni, G.; Fringuelli, F.; Pizzo, F.; Vaccaro, L. Epoxidation of $\alpha, \beta$-unsaturated ketones in water. An environmentally benign protocol. Green Chem. 2003, 5, 425-428. [CrossRef]

54. Ban, L.; Mrksich, M. On-Chip Synthesis and Label-Free Assays of Oligosaccharide Arrays. Angew. Chem. Int. Ed. 2008, 47, 3396-3399. [CrossRef] [PubMed] 